\title{
ELECTRICAL CHARACTERIZATION OF ENGINEERING MATERIALS
}

\author{
MOHAMMAD A. ALIM \\ Hubbell Incorporated, The Ohio Brass Company, 8711 Wadsworth Road, Wadsworth, Ohio 44281
}

(Received September 5, 1995; in final form October 13, 1995)

\begin{abstract}
Engineering material systems for smart components and novel device applications require a thorough understanding on the structure-property-processing relationships to optimize their performance. The factors determining performance characteristics of the multi-phase/component heterogeneous polycrystalline hybrid (MPCHPH) systems are not identical to devices based on single-crystal/single-junction (SCSJ) technology. Performing SCSJ-like data-analysis on the MPCHPH systems can lead to confusion in delineating simultaneously operative phenomena when "physical geometrical factors" are used in normalizing the as-measured electrical parameters or electrical quantities. Such an analytical approach can vitiate interpretation when microstructural inhomogeneity plays a key role in determining the electrical path. The advantage of using the as-measured electrical parameters or electrical quantities constituting the "immittance function" is emphasized. The "state of normalization" using physical geometrical factors can only be executed for a specific phenomenon when isolated from the total electrical behavior.
\end{abstract}

\section{INTRODUCTION}

Many engineering material systems possess highly complex microstructures, which may be regarded as the multi-phase/component heterogeneous polycrystalline hybrid (MPCHPH) materials. Particularly, the structure-property-processing relationships of these systems are crucial in the development of engineering materials for applications as smart components and novel devices. ${ }^{1,2}$ The resulting arrangement of the grains, grain-boundaries, and other phases (when present) constitutes an integrated series-parallel lacy network of conducting paths involving " $m$ " junctions in parallel and " $n$ " junctions in series between the electrode terminals. ${ }^{2,3}$ The lumped behavior of this network indicates dominance of the electrical paths ${ }^{2}$ of the device under test (DUT). In general, a single-crystal/singlejunction (SCSJ) and/or electrochemical-type system is structurally simpler DUT than an MPCHPH based device. Each of these DUTs is subject to engineering applications depending on performance characteristics and appropriate needs.

The ac small-signal immittance (impedance or admittance) data complemented with dc measurement have been utilized in characterizing a variety of electrically active material systems. ${ }^{1-17}$ These measurements are widely exploited as the search of new materials' for various electrical applications continues. In order to extract the phenomena related parameters/elements, the immittance data are displayed in the complex plane formalisms ${ }^{2,17}$ via lumped parameter/complex plane analyses (LP/CPA), which are often supported by the Bode plane analyses ${ }^{8,15}$ (BPA), spectroscopic analyses ${ }^{18,19}$ (SA), etc. These parameters/elements, attributed to the 
conduction processes, can then be used as indicators to optimize microstructural network configuration by varying the chemistry, composition or recipe, processing variables, etc. In addition, these parameters/elements can be used to construct an equivalent circuit model, consisting of the conducting paths, regardless of the complexity in the microstructure. In this way, useful interpretations and conclusions can be drawn for a DUT.

The as-measured "electrical parameters" or "electrical quantities" as a function of frequency $(f)$ can be transformed to the immittance $\left(I^{*}\right)$ function and analyzed in a complex plane formalism. This function represents ac small-signal immittance data in the complex plane formalism by

$$
\mathrm{I}^{*}=\mathrm{I}^{\prime} \pm j \mathrm{I}^{\prime \prime},
$$

where $j=\sqrt{-1}, I^{\prime}$ and I" are the real and imaginary parts of $\mathrm{I}^{*}$, respectively. These real and imaginary parts can be termed as electrical quantities, and each of these parts contain as-measured electrical parameters. The form of the as-measured electrical parameters contained in the electrical quantities depends on the type of the immittance function. The \pm sign also depends on the type of this function. Therefore, the complex plane representation involving the LP/CPA technique employs a plot of $I^{\prime}$ on the $x$-axis (real) versus I" on the $y$-axis (imaginary). In general, the BPA involves an investigation of $I^{*}, I^{\prime}, I^{\prime \prime}, I^{\prime} / I^{\prime \prime}, I^{\prime \prime} / I^{\prime}$, as-measured electrical parameters, etc. as a function of frequency at a specific experimental condition. In the same way, the SA involves similar investigation of these parameters as a sequential function of possible experimental variables, such as variation in temperature, pressure, ambient environment, composition or recipe, fabrication or processing route, voltage etc. As an example, in a parallel configuration of the measurement processes,

$$
\mathrm{I}^{*}=\mathrm{Y}^{*}=\text { admittance }=\mathrm{G}_{p}+j\left(\omega \mathrm{C}_{p}\right)
$$

of the system with

$$
\mathrm{I}^{\prime}=\mathrm{G}_{p}=\text { parallel conductance, and } \mathrm{I}^{\prime \prime}=\omega \mathrm{C}_{p}=\text { parallel susceptance. }
$$

Using the same measurement configuration,

$$
\mathrm{I}^{*}=\mathrm{C}^{*}=\text { capacitance }=\mathrm{C}_{p}-j\left(\frac{\mathrm{G}_{p}}{\omega}\right)
$$

of the system with

$$
\mathrm{I}^{\prime}=\mathrm{C}_{p}=\text { parallel capacitance, and } \mathrm{I}^{\prime \prime}=\frac{\mathrm{G}_{p}}{\omega}=\text { imaginary capacitance. }
$$

In a series configuration of the measurement processes,

$$
\mathrm{I}^{*}=\mathrm{Z}^{*}=\text { impedance }=\mathrm{R}_{s}-j\left(\frac{1}{\omega \mathrm{C}_{s}}\right)
$$


of the system with

$$
I^{\prime}=R_{s}=\text { series resistance, and } I^{\prime \prime}=\frac{1}{\omega C_{s}}=\text { series reactance. }
$$

Using the same measurement configuration,

$$
\mathrm{I}^{*}=\mathrm{M}^{*}=\text { modulus }=\frac{1}{\mathrm{C}_{s}}+j\left(\omega \mathrm{R}_{s}\right)
$$

of the system with

$$
\begin{aligned}
& I^{\prime}=\frac{1}{C_{s}}=\text { inverse series capacitance (real modulus), and } I^{\prime \prime}=\omega R_{s} \\
& =\text { imaginary modulus. }
\end{aligned}
$$

Here, $G_{p}$ and $C_{p}$ are the as-measured parallel equivalent terminal conductance and capacitance, respectively, and $R_{s}$ and $C_{s}$ are the as-measured series equivalent terminal resistance and capacitance, respectively, as a function of angular frequency $\omega(=2 \pi f)$. These four as-measured (terminal) electrical parameters (i.e., $G_{p}, C_{p}, R_{s}$, and $\mathrm{C}_{\mathrm{s}}$ ) are the basic constituents of the immittance function.

The "state of normalization" using "physical geometrical factors" in the immittance data can be traced back to a pioneering work by Cole and Cole. ${ }^{20}$ They highlighted non-Debye (non-ideal) electrical behavior in a single relaxation process, and demonstrated an analytical approach of the immittance data via a complex plane formalism $\left(\mathrm{C}^{*} \equiv \epsilon^{*}\right)$. As the total electrical response exhibited SCSJ-like behavior, the state of normalization did not affect the fundamental aspect of the demonstration. Grant ${ }^{21}$ applied this approach further to polycrystalline materials exhibiting single-like relaxation process. He established simultaneous multiple complex plane analysis (i.e., $\mathrm{C}^{*} \equiv \epsilon^{*}$ ) and $\mathrm{Y}^{*} \equiv \sigma^{*}$ ) of the same immittance data, and demonstrated multiple aspects of the dielectric representation of a DUT. The parameters $\epsilon^{*}$ and $\sigma^{*}$ are the normalized representation of $C^{*}$ and $\mathrm{Y}^{*}$, respectively, using physical geometrical factors. Their significance is discussed in section $\mathbf{V}$. The physical geometrical factors refer to the physical electrode area and physical thickness of the DUT.

Grant's demonstration inspired many investigators to use the state of normalization in polycrystalline materials regardless of the number of relaxation processes in a complex plane formalism. Such an approach should be adopted with caution when microstructural lacy network plays a key role in determining electrical paths. This is because it restricts significance of specific terminology, i.e., nomenclature for every isolated phenomenon, and does not portray the same situation as it does for a homogeneous system. In other words, an approach involving the use of physical geometrical factors may give rise to erroneous interpretations when applied to a non-SCSJ system. In view of escalating state of normalization using physical geometrical factors in the literature, this paper will suggest a simple and universal 
way of handling/analyzing the as-measured immittance data for all types of DUTs so that the electrical response can be evaluated without leading to erroneous conclusions.

In this paper, the problems associated with the state of normalization of the as-measured immittance data are discussed. Relevant issues concerning multiple relaxations using the LP/CPA technique, especially the nomenclature used in establishing operative phenomena, are highlighted. Possible detrimental aspects of short frequency range immittance measurements (such as single- or spot-frequency datum) on the interpretation are emphasized. Cumulative dividends of the corrective approaches addressing these problems are suggested based on effective data-handling/analyzing criteria. A detailed description of the analytical approaches, and the extracted parameters in conjunction with the derived units of the electrical parameters or quantities (wherever appropriate for the as-measured/ terminal parameters including real and imaginary parts of $I^{*}$ ) are also provided. It will be demonstrated that the suggested data-handling/analyzing criteria can yield fruitful results regardless of the degree of microstructural heterogeneity within a DUT. Some generic schematics and results of the multi-plane analytical approaches of several material systems are given to illustrate the main theme of this paper. ${ }^{2,3,17,22}$

\section{BACKGROUND: "SCSJ” AND “MPCHPH” SYSTEMS}

An MPCHPH system cannot be treated like an SCSJ system as it cannot be represented by either a uniform or homogeneous configuration. Under certain limiting conditions an MPCHPH system can exhibit SCSJ-like behavior. A thorough understanding on these limiting conditions is necessary to deleneate the demarcation between the SCSJ-like response and MPCHPH behavior. This demarcation can be evaluated using total response in the frequency-domain under non-equilibrium experimental conditions. Such an evaluation may indicate overlapped mechanisms due to non-distinguishable successive semicircular relaxations and severe distortion in the relaxation processes in a complex plane formalism. $^{10,13,22}$

In an MPCHPH-type DUT, the current flows through the easiest paths consisting of the series-parallel microstructural network. In other words, the electrical conduction processes occur along the paths which are the shortest between the electrode terminals. Thus, the grains and other relevant phases must produce lumped series effect with the grain-boundaries, as the inter-connecting junctions parallel (or nearly parallel/horizontal) to the physical electrode surface are not likely to be the shortest electrical path. This situation can be visualized from Figure 1(a) where variation in microstructural grain-orientation is considered. The series equivalent conducting path, assumed to be perpendicular (or nearly perpendicular/ vertical) to the electrode surface, must be operative between the two-terminal electrodes. For all practical purposes, possible interference from the cross-linked series-parallel lacy network within the microstructure ${ }^{1}$ may be assumed to be negligible, and can have either no or very weak effect on the parallel conducting 


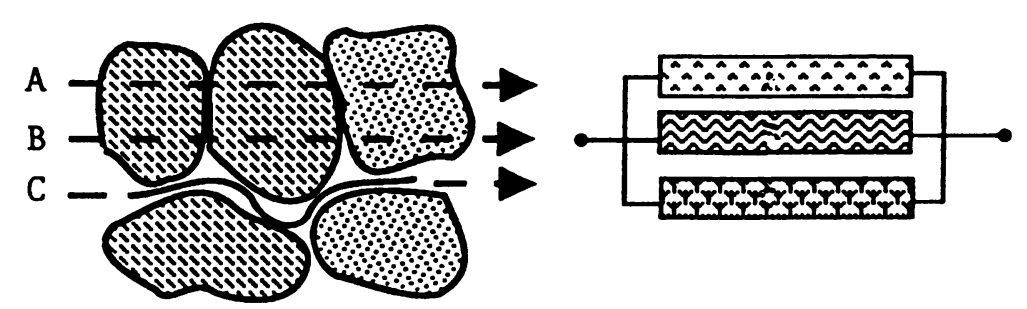

a. Each significant conduction path must be represented by a parallel equivalent circuit path.

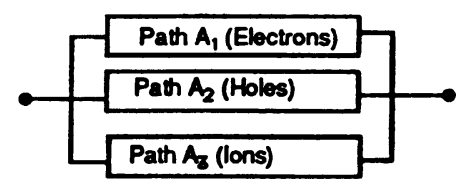

b. Each significant charge carrying species must be represented by a parallel equivalent path.

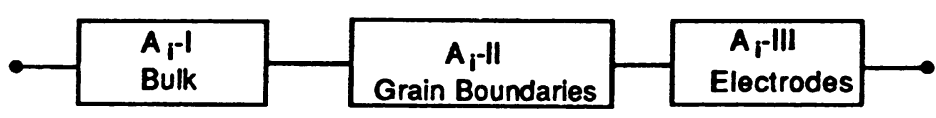

c. Each region of significance within a path must be represented by a lumped parameter circuit with $l=1,2$ and 3 (Figure b) for the type of charge carriers.

FIGURE 1 Illustration of the lumped representation incorporating simultaneously operative competing phenomena in an electrically active multi-phase/component heterogeneous polycrystalline system.

paths simultaneously operative between the two-terminal electrodes. Each contributing carrier in the lumped grains constitute parallel combination within an electrical path as shown in Figure 1(b). Additional contribution from the electrode material and its possible interaction with the DUT must be in series with the lumped series behavior of the grains, grain-boundaries, and other phases.

The physical location of each of these microstructural entities between the measurement terminals contributes to the net series effect, as demonstrated in Figure 1(c). Thus, Figure 1 illustrates the lumped representation incorporating simultaneously operative competing phenomena in an electrically active MPCHPH system.

In addition to the foregoing discussion on the microstructural conduction modeling, a serious attention is required in the immittance data analyses when the porosity becomes a key issue. In the event an MPCHPH system (such as ceramic-based gas sensing materials ${ }^{7}$ ) needs a finite degree of porosity in optimizing performance characteristics, a number of factors must be monitored carefully. 
These include physical geometrical factors, grain-to-grain contact area, currentvoltage data, dc superimposed ac small-signal response (i.e., capacitance versus voltage or Mott-Schottky ${ }^{22}$ representation), frequency range of the immittance data, status of other non-equilibrium experimental variables, etc. As the degree of porosity approaches zero (i.e., achieving nearly theoretical density), the frequencydomain response may differ greatly from that obtained in the highly porous state. This is expected because the physical contact area across the grain-boundary starts to vary significantly. Evidently, physical processes causing net contribution(s) to the conducting path(s) can be influenced by the variation in the type of planer/nonplaner contact area across the grain-boundary. For practical purposes a DUT of near theoretical density ( $\approx 99 \%$ or better) is less critical and, thus, its influence on the electrical measurements can be neglected. However, for a small change in the porosity, the frequency-domain response may be reproduced as the operative mechanisms remain the same for either dense or porous system.

The foregoing discussion does not exclude an electrochemical-type DUT, as the analyses of the immittance data are not different from those of the SCSJ and MPCHPH systems. Also, the data-handling/analyzing criteria, which will be described later, are the same for an electrochemical system regardless of its origin and the type of ac small-signal response.

\section{ELECTRICAL REPRESENTATION OF COMPETING PHENOMENA}

It is convenient to represent a DUT in the form of a lumped equivalent circuit. This is a general practice for the SCSJ-type DUTs, such as $p$ - $n$ junctions, metalsemiconductor diodes, $p-n-p / n-p-n$ transistors, MOS/MIS and MOSFET/MISFET devices, etc. Some general illustrations are given in Figure 1 to represent simultaneously operative competing phenomena occuring within a DUT. These phenomena can be represented by an equivalent circuit model. This model is developed by following a set of rules ${ }^{2,17}$ summarized below:

1. each significant conduction path must be represented by a parallel equivalent circuit path (Figure 1a);

2. each significant charge carrying species must be represented by a parallel equivalent path (Figure 1b);

3. each region of significance within a path must be represented by a lumped parameter circuit with respect to the type of charge carrying species (Figure 1c);

4. each mechanism of polarization and relevant phenomena must be represented by either a series or parallel combination of resistors, capacitors and/or inductors (inductive effect is excluded in the subsequent discussion);

5. the components representing various conducting species and polarization processes each has its own composition, microstructural, temperature, voltage, pressure, environment, and frequency dependence;

6. the electrical paths of significance are those yielding the least impediment to current flow; 
7. the regions of significance within these electrical paths are those presenting the largest impediment to current flow; and

8. analytical techniques (such as exploitation of the complex plane formalisms in conjunction with the Bode plane analysis and spectroscopic plots) can be used to elucidate an appropriate equivalent circuit representation from measured immittance components when used concurrently with the information obtained from other methods.

Even with the application of these rules, difficulties may arise for a complicated DUT system in which the electrical paths exhibit multiple current or voltage dividing lumped segments. Each of these segments may not necessarily represent SCSJ-like behavior. For example, "bulk phenomenon" may suit SCSJ-like representation only when it is isolated from all other extraneous contributions, and only then the state of normalization can be applied. Should other simultaneously operative phenomena, such as trapping within grain-boundaries and depletion regions, resonating behavior, charge accumulation effect at the DUT-electrode interfaces, electrode contribution or polarization from work-function mismatch, etc., demand an SCSJ-like representation, an adequate evaluation process of the total characteristics is necessary. In the following sections it will be shown how the state of normalization cannot be adopted as a universal route for data-analysis and subsequent interpretation.

\section{EFFECT OF "STATE OF NORMALIZATION"}

A comprehensive understanding on the nature of the dispersion of the immittance data with frequency is necessary before normalizing the as-measured electrical parameters or quantities. This helps resolving the total behavior of a DUT. Upon identifying each contributing phenomenon, necessary parameters to be extracted can be outlined with a meaningful interpretation, and carefully examined to warrant the adequacy of the state of normalization using the physical geometrical factors for the particular parameters. The state of normalization can only be applied to the those parameters which are truly associated with the physical processes within a DUT. In other words, the generalization of the state of normalization by incorporating physical geometrical factors before performing analysis is inappropriate due to at least for the following reasons:

1. operative phenomena between the electrode terminals are unknown;

2. multiple relaxation processes may not to be distinct (possible overlapped and/or distorted behavior);

3. multiple operative phenomena are not distinguishable from a single lumped relaxation process [requires further analytical tool(s)/technique(s) to delineate];

4. limitation of the analytical tools/techniques and window of visibility are uncertain;

5. contribution of each phase to the total relaxation processes is unknown; 
6. contact area for each contributing electrical path between the successive grains is unknown;

7. statistical variation in the contact areas (across a junction) is uncertain;

8. cross-section of effective contribution within each contact area is unknown (entire contact area does not necessarily contribute to the total conduction due to planer/non-planer situation, not all of the contact area is involved in many DUTs);

9. distribution (spatial or localized) of traps is unknown; and

10. distribution of electrical barrier voltages is unknown.

These reasons may simultaneously exist in DUTs exhibiting multiple relaxations in one or more complex plane or a single relaxation in one complex plane. For multiple relaxations in one complex plane:

1. admixture of Debye and non-Debye responses influencing each operative electrical path is unknown;

2. admixture of multiple non-Debye responses influencing each operative electrical path is unknown; and

3. resulting non-Debye response for each relaxation is unlikely to originate from the ordinate parameter (non-Debye behavior is attributed to the depression of the ordinate parameter).

For a single relaxation in one complex plane:

1. range of frequency spectrum (measurement range as a function of frequency) is likely to be limited;

2. resulting Debye or non-Debye relaxation is likely to be lumped (possible for a Debye relaxation, common for a non-Debye relaxation) so that distinction is not possible (described in 3); and

3. ordinate parameter remains critical for the non-Debye response as it does for multiple relaxations.

Therefore, a single-frequency (designated as spot-frequency) immittance dataanalysis incorporating physical geometrical factors of the DUT may lead to erroneous or physically meaningless conclusions. In other words, it is too much to speculate about the mechanism(s) for the pair of normalized real and imaginary immittance components corresponding to a single-frequency. Judgement based on the single-frequency immittance data can be valid only when the entire frequencydomain response is thoroughly understood through a systematic evaluation process. This frequency-domain response assures a total dispersion characteristic as a function of frequency, and can be significantly influenced by the presence of several types of distinguishable or non-distinguishable traps. These traps are characterized $^{3,21-23}$ by the energy depth, capture cross-sectional area, defect charge state (donor/acceptor-like, neutral, etc.), capture-rate constituting elements (resistance $\mathbf{R}$ and capacitance $C)$ of the relaxation time $(\tau=R C)$, etc. When the frequencydomain response of the simultaneously operative phenomena are not explored, the underlying physical process(es) of an unknown DUT remains unresolved. 


\section{DATA-HANDLING/ANALYZING CRITERIA}

In order to establish a meaningful approach to the data-handling criteria, attempts have been made by several investigators. ${ }^{2-17,22}$ on a number of material systems. These attempts employed a series of systematic evaluation ascertaining uniform geometry (i.e., possessing identical physical geometrical factors) and a consistent history in the microstructural features of the DUTs. In this section the acquisition and handling/analyzing procedure of the dc data are excluded, as it is a special case with the ac small-signal measurement for $f \rightarrow 0 \mathrm{~Hz}$. The frequency-domain measurements can be accomplished via the acquisition of the two-terminal ac small-signal electrical data as a function of frequency in one of the following three forms ${ }^{2,17}$ :

1. impedance $\left(\mathrm{Z}^{*}\right)$ or series equivalent (a resistor, $\mathrm{R}_{\mathrm{s}}$, in series with a capacitor, $\mathrm{C}_{\mathrm{s}}$ );

2. admittance $\left(\mathrm{Y}^{*}\right)$ or parallel equivalent (a resistor, $\mathrm{R}_{\mathrm{p}}$ or a conductor, $\mathrm{G}_{\mathrm{p}}$, in parallel with a capacitor, $\mathrm{C}_{\mathrm{p}}$ ); and

3. $\left|\mathrm{Z}^{*}\right|$-phasor or $\left|\mathrm{Y}^{*}\right|$-phasor (magnitude of immittance and the corresponding loss-tangent, $\tan \delta$, or phase angle, $\delta$ ).

The measurements are equally useful in any of these three forms, and can be referred to as the immittance data described earlier. These three forms are illustrated in Figure 2. The choice depends on the material characteristics and the resolution of the measuring parameters in a particular frequency range. A trial run of the acquisition may be helpful before the final data are acquired. This step allows an initial judgement on the dispersion of immittance with frequency. Usually the value of $\tan \delta$ dictates the degree of resolution in the measuring parameters. To reduce the stray and lead effects it is better to keep shorter contact leads.

The amplitude of the applied ac signal must be adjusted, without affecting intrinsic immittance response, to obtain satisfactory data. Reproducibility of the data depends on sufficient modulation of the quasi-equilibrated Fermi-level of the DUT. The measurement range of frequency is independent of the small-signal amplitude. When the signal amplitude is too low, the immittance data may contain mainly noise. The noise may represent discrete or inconsistent pattern in the data and appear as scattered points in the plot. Therefore, an adjusted amplitude of the applied ac signal is crucial.

The polarization effects at low and ultra-low frequencies (usually in the $\mathrm{Hz}$ and sub-Hz regions) also yield noise and data-scattering despite reasonable amplitude of the ac signal. Under these circumstances, instead of increasing the amplitude of the ac signal, enough equilibrium time may be allowed to obtain satisfactory data. On the other hand, at sufficiently high amplitude of the ac signal, the terminal immittance may be affected by the geometric effect within the DUT in addition to the modulation of the quasi-equilibrated Fermi-level. The utilization of the fast Fourier transform (FFT) in conjunction with averaging the measured electrical parameters or quantities for a suitably fixed number of observations within a specified time is a common practice to avert such problems. ${ }^{2,3,22}$ The use of FFT is a standard practice for measurements at ultra-low frequencies in the sub- $\mathrm{Hz}$ region. 


\section{A. Preferred Measured Forms}

(a) Series:

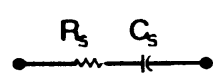

(b) Parallel:

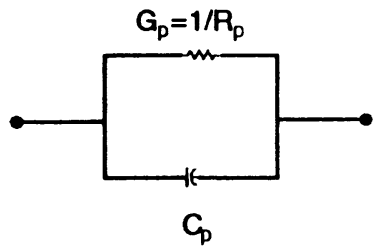

(c) Phasor: $\left|Z^{*}\right|$ or $I Y^{*} \mid$ and loss tangent $\left(\tan \delta=G_{p} / \omega C_{p}=\omega R_{s} C_{s}\right)$

B. Eour complex Plane Equations $\left(\omega=2 \pi f, f=\right.$ measured frequency, $j=(-1)^{1 / 2}$ )

1. Impedance: $Z^{*}=R_{s} \cdot j\left(1 / \omega C_{s}\right)$

2. Admittance: $Y^{*}=G_{p}+j \omega C_{p}$

3. Capacitance: $C^{*}=C_{p}-j\left(G_{p} / \omega\right)$

4. Modulus: $\quad M^{*}=1 / C_{s}+j \omega R_{s}$

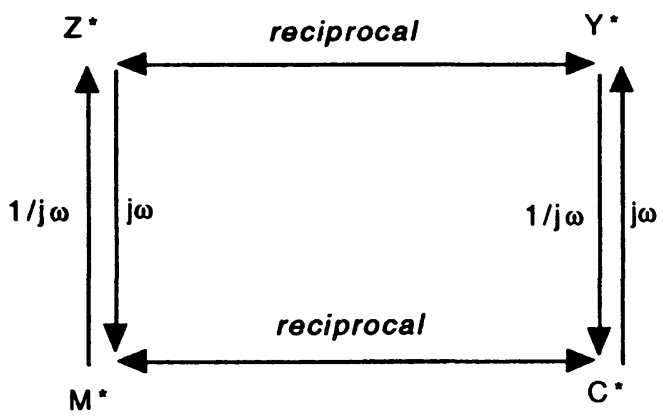

Fundamental equations in four complex planes representing the measured form of data and its transformation related to electrical characteristics consisting of $Z^{*}, Y^{*}, C^{*}$ and $M^{*}$. Arrows imply multiplying factors for conversion between the complex planes.

FIGURE 2 Choice of measured forms of the ac small-signal electrical data acquisition and inter-convertible relationships within the four complex plane formalisms.

The averaging procedure could utilize self-developed measurement processes without employing FFT (generally in the $\mathrm{Hz}$ region but below $\mathrm{kHz}$ ) which reasonably satisfy the reproducibility of the data-acquisition method at low frequencies. This is a beneficial approach to certain DUTs when the low-frequency measurement becomes essential. 
The admittance $\left(\mathrm{Y}^{*}\right)$ and impedance $\left(\mathrm{Z}^{*}\right)$ obtained as a function of the ac small-signal frequency, consisting of parallel and series parameters operative in-phase (constituting real part) and out-of-phase (constituting imaginary part), respectively, are expressed in equations 2 and 6 . The phasor data also translate to either form of these two equations. As an example, the phasor form may be expressed for $\mathrm{Z}^{*}$ or $\mathrm{Y}^{*}$ as:

$$
\mathrm{Z}^{*}=\frac{1}{\mathrm{Y}^{*}}=\left|\mathrm{Z}^{*}\right| \sin \delta+j\left|\mathrm{Z}^{*}\right| \cos \delta=\left|\mathrm{Z}^{*}\right| e^{j \delta}=\frac{\mathrm{V}}{\mathrm{I}} \angle \delta
$$

where $\left|Z^{*}\right|$ is the absolute magnitude of $Z^{*}, \delta$ is the phase angle between the voltage, V, across, and the current, I, through the DUT. Thus, these three forms of data acquisition are equivalent and serve the same purpose. Subsequent analysis using any form of these frequency-domain data can be performed via:

1. lumped parameter/complex plane analyses;

2. Bode plane analyses; and

3. spectroscopic analyses.

Despite using these three analytical techniques in a simultaneous fashion, the underlying physical processes for a complicated DUT system may not be unraveled. Usage of additional tool(s)/technique(s) may be necessary to resolve complicated situations. One of the techniques known as deep-level transient spectroscopy (DLTS), not included here, employs the time-domain measurements. ${ }^{25}$ It is worth pointing out that both the frequency-domain and time-domain measurements can be regarded as a time-zone process yielding complementary results.

\section{Lumped Parameter/Complex Plane Analyses}

To perform the LP/CPA, the data in any form of the immittance function can be used. The nature of the operative electrical path is facilitated by displaying these data in the form of complex plane plots. A trial analysis in four complex planes is necessary for the as-measured immittance data. Each of these complex planes can represent a DUT with particular characteristics. These plots, when combined with other known features of the DUT, allow the representation of an appropriate equivalent circuit model. This model can be used to delineate the contribution of various regions (of the microstructure) with respect to the overall electrical behavior of the DUTs between the electrode terminals, including the influence of process variables on these regions. These four complex planes are inter-convertible as shown in Figure 2. By incorporating the physical geometrical factors, each of these four complex plane formalisms can be described below with appropriate nomenclature, symbol, equivalent synonym, and one consistent unit. These are:

1. impedance $\left(Z^{*}=Z^{\prime}-j Z^{\prime \prime}\right)$ in $\Omega(o h m)$, or resistivity ( $\left.\rho^{*}=\rho^{\prime}-j \rho^{\prime \prime}\right)$ in $\Omega$-cm (ohm-cm);

2. admittance $\left(\mathrm{Y}^{*}=\mathrm{Y}^{\prime}+j \mathrm{Y}^{\prime \prime}\right)$ in $\mathrm{S}$ (siemen or mho), or conductivity $\left(\sigma^{*}=\sigma^{\prime}\right.$ $\left.+j \sigma^{\prime \prime}\right)$ in $\mathrm{S} / \mathrm{cm}(\mathrm{mho} / \mathrm{cm})$; 
3. capacitance $\left(\mathrm{C}^{*}=\mathrm{C}^{\prime}-j \mathrm{C}^{\prime \prime}\right)$ in $\mathrm{F}$ (Farad), or permittivity $\left(\epsilon^{*}=\epsilon^{\prime}-j \epsilon^{\prime \prime}\right)$ in $\mathrm{F} / \mathrm{cm}$; and

4. modulus $\left(\mathrm{M}^{*}=\mathrm{M}^{\prime}+j \mathrm{M}^{\prime \prime}\right)$ in $\mathrm{F}^{-1}(1 /$ Farad $)$, or electric-modulus $\left(m^{*}=m^{\prime}\right.$ $\left.+j m^{\prime \prime}\right)$ in $\mathrm{F}^{-1}-\mathrm{cm}$.

The first group of the complex plane formalisms, consisting of $\mathrm{Z}^{*}, \mathrm{Y}^{*}, \mathrm{C}^{*}$, and $\mathrm{M}^{*}$ plots, is constructed from the as-measured electrical parameters shown in Figure 2. The second group of the complex plane formalisms, consisting of $\rho^{*}, \sigma^{*}, \epsilon^{*}$, and $m^{*}$ plots, employed the state of normalization using the physical geometrical factors. The direction of vertical arrows (upward and downward) in Figure 2 indicates the multiplication factor $j \omega$ or $1 / j \omega$ to be used for the conversion between the associated pair of complex planes. The horizontal arrows indicate reciprocal relationship between the relevant pair of complex planes. The inter-convertible relationships among these complex planes exist in the following way:

$$
\begin{aligned}
& \mathrm{Z}^{*}=\frac{1}{\mathrm{Y}^{*}}=\frac{\mathrm{M}^{*}}{j \omega}=\frac{1}{j \omega \mathrm{C}^{*}}=\mathrm{R}_{s}-j\left(\frac{1}{\omega \mathrm{C}_{s}}\right), \\
& \mathrm{Y}^{*}=\frac{1}{\mathrm{Z}^{*}}=j \omega \mathrm{C}^{*}=\frac{j \omega}{\mathrm{M}^{*}}=\mathrm{G}_{p}+j\left(\omega \mathrm{C}_{p}\right), \\
& \mathrm{C}^{*}=\frac{1}{\mathrm{M}^{*}}=\frac{\mathrm{Y}^{*}}{j \omega}=\frac{1}{j \omega \mathrm{Z}^{*}}=\mathrm{C}_{p}-j\left(\frac{\mathrm{G}_{p}}{\omega \mathrm{C}_{p}}\right),
\end{aligned}
$$

and

$$
\mathrm{M}^{*}=\frac{1}{\mathrm{C}^{*}}=j \omega \mathrm{Z}^{*}=\frac{j \omega}{\mathrm{Y}^{*}}=\frac{1}{\mathrm{C}_{s}}+j\left(\omega \mathrm{R}_{s}\right)
$$

The dimensionless relative permittivity

$$
\epsilon_{r}^{*}=\epsilon_{r}^{\prime}-j\left(\epsilon_{r}^{\prime \prime}\right)
$$

may be derived from

$$
\epsilon^{*}=\epsilon^{\prime}-j\left(\epsilon^{\prime \prime}\right)
$$

incorporating $\epsilon_{0}\left(\approx 8.85 \times 10^{-14} \mathrm{~F} / \mathrm{cm}\right)$. This dimensionless complex quantity provides the same information as the $\mathrm{C}^{*}$ - or $\epsilon^{*}$-plane plot.

The sample plots for each of the four complex plane formalisms are depicted in Figure 3. Each plot contains a simplified equivalent circuit representing an intercept on the left-side of the single semicircular relaxation on the $x$-axis. This intercept may be termed as the left-intercept. The ordinate ( $y$-axis) and abcissa ( $x$-axis) must have the same plotting scale so that the semicircular relaxation in any of these complex planes can be clearly delineated. This means that the magnitude of each unit grid 

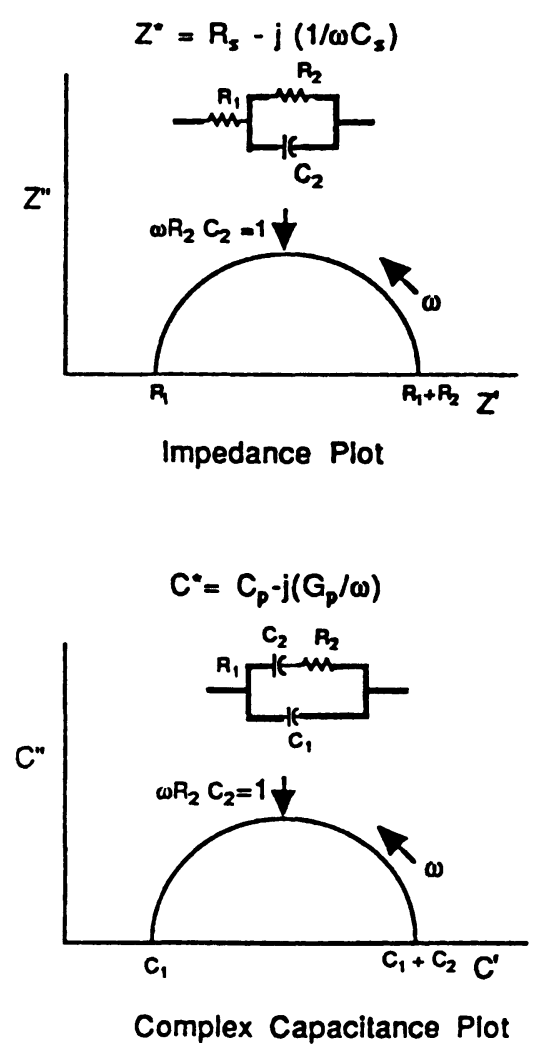
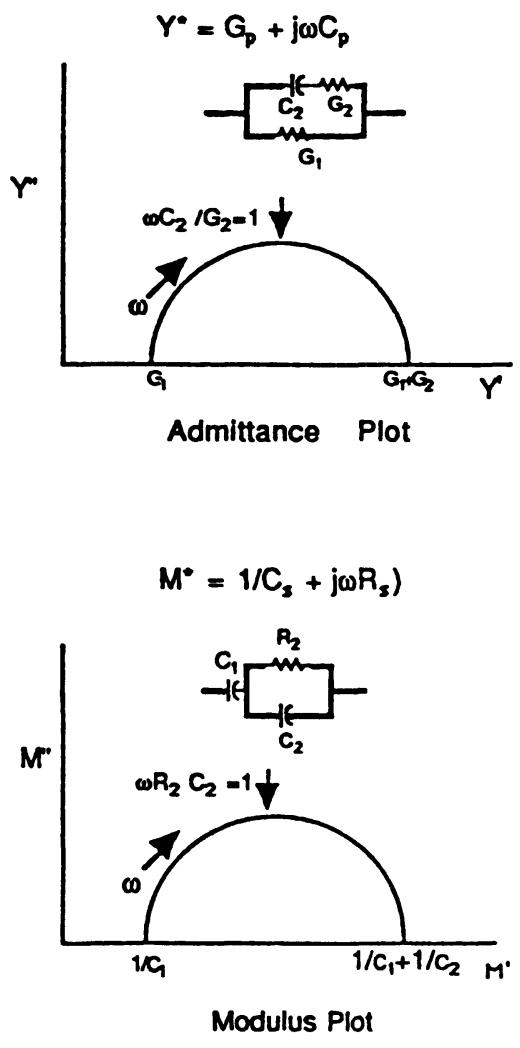

FIGURE 3 Sample plots with associated simplified equivalent circuits for each of the four complex planes.

(or graphical segment) in length on ordinate and abcissa must be equal. A semicircle whose center lies on the $x$-axis is defined as a Debye or Debye-like relaxation. The transformation from impedance to admittance or vice versa is straightforward only for the Debye relaxation. For a non-Debye relaxation, of which the center of the semicircle lies below the $x$-axis, an empirical relation ${ }^{20}$ is available for such a transformation. A non-Debye relaxation gives rise to a depression angle $(\theta)$ measured from the point at the left-intercept to the center of the semicircle below the $x$-axis. This depression angle becomes a measure of the depression parameter, $h$, where $h=2 \theta / \pi$. Depending on the idealized Debye or extreme non-Debye response of a semicircular relaxation the value of $h$ ranges between 0 and 1 . Thus, a Debye-like behavior can be achieved as $\theta \rightarrow 0^{\circ}(h \rightarrow 0)$, and an extreme non-Debye response can be visualized with $\theta \rightarrow 90^{\circ}(h \rightarrow 1)$.

The aforementioned nomenclatures of each group of the four complex plane formalisms are widely used. Using the same nomenclature, possessing a unit other than mentioned above, can distort the meaning of the plot when multiple semicircular relaxations are observed in one complex plane. This is discussed in section VII. It is important to note that with growing popularity of the immittance measurements and subsequent analyses, a vague nomenclature referring to 
"Nyquist diagram" or "Nyquist plot" is used for a complex plane formalism. Particularly, the plot of real and imaginary parts of impedance or admittance are described with this nomenclature. Macdonald ${ }^{26}$ has discouraged the use of such a vague nomenclature and pointed out that immittance measurements have no connection with the original work of Nyquist. He elaborated on the fact that the immittance is derived from the measurements of current and potential at a single (input) port recognizing a phase difference for a real-world DUT. ${ }^{1}$ However, Nyquist $^{26}$ dealt with two-port measurements of feedback in amplifiers which involved input and output voltage determinations. Thus, Macdonald reasonably argued that a complex plane "Nyquist plot" is intrinsically quite different from an immittance function (I*) plot.

From the nature of acquisition of the in-phase and out-of-phase electrical parameters or quantities, each complex plane formalism intrinsically represents a complex quantity possessing real and imaginary parts. Therefore, the four complex plane formalisms do not need to be emphasized with a prefix complex. Also, since the immittance data acquisition employs the ac small-signal, there is no need of stressing impedance by ac impedance. Similar argument applies to other complex plane formalisms. To avoid ambiguity between a real and a complex quantity, the four complex plane formalisms can be denoted by a superscript asterisk, such as $\mathrm{Z}^{*}$, $\mathrm{Y}^{*}, \mathrm{C}^{*}$, and $\mathrm{M}^{*}$.

The unit of the constituting components for each immittance function (such as "resistance and reactance" for $\mathrm{Z}^{*}$ and "conductance and susceptance" for $\mathrm{Y}^{*}$ ) should be used with caution. If the chosen unit is derived to serve a particular purpose, the reasons of using the derived unit should be justified. It is necessary to emphasize the significance of the derived unit for the constituting components. Thus, a unit should refer to a meaningful physical parameter responsible for the immittance function. As an example, the capacitance per unit area or conductance/ resistance per unit area should be considered at least for two reasons: (1) applicability to the material system, and (2) selectivity of the series or parallel configuration for the data-analyses. Only when these two reasons are justified, the complex plane formalism with the derived unit on the axes is meaningful. Extending the concept further, series configuration (i.e., impedance representation of the acquired data) does not resolve the blocking situation of an $\mathrm{R}_{\mathrm{s}}-\mathrm{C}_{\mathrm{s}}$ circuit under $\mathrm{dc}$ $(f \rightarrow 0 \mathrm{~Hz})$ condition. The parallel configuration (i.e., admittance representation of the acquired data) of an $R_{p}-C_{p}$ circuit does not portray the same situation as the $R_{s}-C_{s}$ series combination satisfying $R_{p}$ equal or equivalent to $R_{s}$ and $C_{p}$ equal or equivalent to $\mathrm{C}_{\mathrm{s}}$.

The major part of the data-handling criteria involves proper fitting of the semicircular relaxation. Due to the lack in precautions in fitting the semicircular loci, erroneous interpretation is possible. The degree of accuracy in fitting the actual data points on the semicircular loci is achieved by using the complex nonlinear least squares (CNLS) analyses. ${ }^{27-34}$ This approach ascertains proper numerical values of the extracted parameters described in section VI.

Complexity arises if a single semicircular relaxation (with or without "leftintercept") is observed in more than one complex plane for the same set of data. This kind of representation is referred to multi-plane analytical approach. The 
circuit(s) derived from the multi-plane analyses may either represent more lumped or resolved behavior. The complexity of multi-plane representation can be delineated with the help of information on processing variables, microstructural features, phase distribution, role of dopants/additives, nature of the electrical barriers, nature of dispersion of the measured parameters in a frequency range for overlapped response(s) between the successive operative phenomena, etc. The investigator has to determine the root or origin of the multiple complex plane representation of the data. Once the root is determined, the interpretation is as straight-forward as a single-plane representation of the data. The conclusion is left to the investigator as only one valid interpretation with an equivalent circuit model is appropriate for a DUT. Further, the multi-plane response may influence the electrical parameters set for the applications criteria of a DUT.

A higher degree of complexity is encountered in DUTs having multiple semicircular relaxations ${ }^{2-7,17,22}$ in more than one complex plane. In such cases, it is found that the series circuits (i.e., voltage dividing or series segments) are best represented by the $Z^{*}$ - and $\mathbf{M}^{*}$-plots, while parallel circuits (i.e., current dividing or parallel segments) are best represented by the $\mathrm{Y}^{*}$ - and $\mathrm{C}^{*}$-plots. The relative magnitudes of various circuit elements govern the choice between the $Z^{*}$ - and $\mathrm{M}^{*}$ or between the $\mathrm{Y}^{*}$ - and $\mathrm{C}^{*}$-plane formalisms. The complexity of the systems that can be analyzed using LP/CPA is exemplified in references 2 and 17. The results of some case studies and limiting factors are also listed there.

Multiple relaxations in one complex plane and their simultaneous representation in other complex plane(s) make the situation even more complicated. ${ }^{2-7,22}$ Such a situation is very common in the MPCHPH systems (such as $\mathrm{ZnO}$-based varistors, ${ }^{3,22,35} \mathrm{TiO}_{2}$-based gas sensing materials, ${ }^{7,36} \mathrm{Y}_{2} \mathrm{O}_{3}$ - and $\mathrm{CaZrO}_{3}$-based materials $^{5,6}$, etc.). Nevertheless, a systematic analytical approach, involving a careful microstructural evaluation in conjunction with consistent thermal or processing history of the DUTs, can provide an adequate solution as recently demonstrated. ${ }^{5-7}$

\section{Bode Plane Analyses}

The classical BPA involves plotting of the absolute magnitude of the immittance function as a function of frequency. ${ }^{24}$ It can provide further understanding on the behavior of the phase angle, as-measured electrical parameters, in-phase (real) and out-of-phase (imaginary) quantities of the immittance function, etc. From the shape of the curve on each Bode plane, an equivalent circuit analog may be developed. In simplified cases, this approach can reveal results identical to that obtained from the LP/CPA. The time-dependent behavior and/or reaction-related dynamic processes are, however, generally not straightforward in the LP/CPA representation. The response is usually distorted and/or overlapped with the other mechanism(s), and often cannot be resolved due to severely lumped behavior of the simultaneously competing phenomena. ${ }^{3-7,22}$ In this case, extraction of an equivalent circuit in a simplified form is not meaningful as it may not match with the information revealed via the LP/CPA technique. This situation can be improved using spectroscopic analyses discussed in the following sub-section. 
Spectroscopic Analyses

The SA consists of sequential analytical steps of the as-measured immittance components (or phasor form), under the non-equilibrium experimental conditions (such as applied voltage, temperature, compositional change, processing variables, ambient environment, pressure, etc.). It has been used by several investigators. $^{3-7,18,19,22,23}$ This analytical approach is useful in the cases where the measurement window is limited due to the lack of proper instrumentation. For example, if the relaxation process is not visible in the complex plane representation at room temperature, the spectroscopic approach as a function of temperature can be useful and yield results identical to those obtained from the LP/CPA or BPA technique..$^{3-7,22}$ Figure 4 illustrates such a correspondence of these two analytical methods. The spectroscopic technique can be very effective especially when a DUT does not yield a meaningful solution in the LP/CPA and BPA, as it involves the evaluation of the variation in the as-measured in-phase and out-of-phase parameters (series or parallel configuration) under non-equilibrium conditions.

Measured form : $Y^{\bullet}=G_{p}+j \omega C_{p}$

Admittance Spectroscopy

$\omega_{x}\left(=1 / \tau_{x}\right)$ is constant

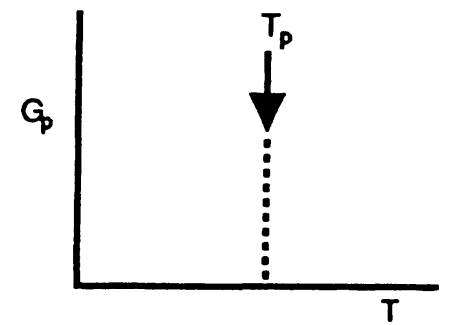

$T_{p}=$ Temperature where $G_{p}$ is maximum at a frequency $\boldsymbol{\omega}_{x}$

$T_{x}=T=$ Measured Temperature

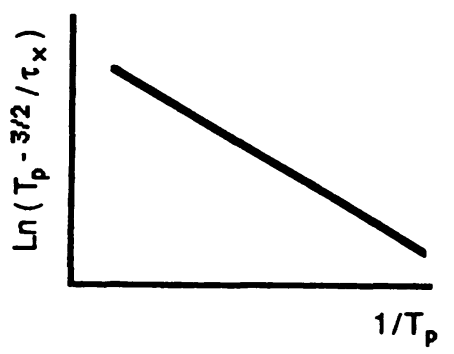

Lumped Parameter/Complex Plane Analysis

$T_{x}$ is constant

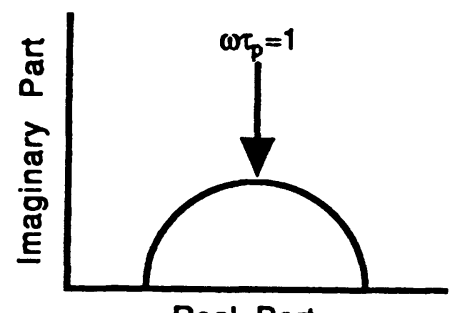

Real Part

Complex Plane Plot

FIGURE 4 Demonstration of identical results obtained via lumped parameter/complex plane analysis (LP/CPA) and spectroscopic analysis (SA) techniques.

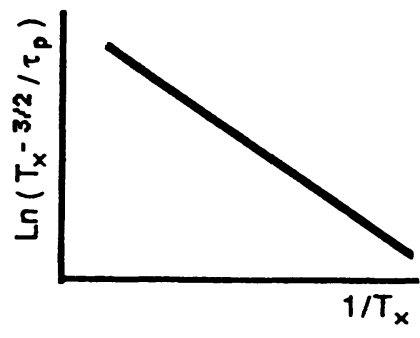




\section{EXTRACTION OF PARAMETERS}

The equivalence of the complex plane formalisms, Bode plane analyses, and spectroscopic plots has been well established. ${ }^{2-24}$ Therefore, for a single Debye/ non-Debye relaxation phenomena the introduction of "physical geometrical factors" in these analytical techniques may not be too critical. From the frequencydomain analytical consideration, several electrical parameters of a DUT can be extracted using these techniques. These parameters are very useful for the optimization of the device characteristics and resulting performance. In general, the common interest for an electrical characterization involves extraction of the following parameters: ${ }^{2,37}$

1. voltage or current dividing equivalent circuit;

2. equivalent circuit elements (resistor, capacitor, inductor, etc.);

3. relaxation time (time-constant or peak-frequency);

4. left and right intercepts on the real axis for each semicircular relaxation;

5. chord or diameter (chord represents a depressed semicircle) of a semicircular relaxation;

6. depression angle $(\theta=h \pi / 2)$ or depression parameter $(0 \leq h \leq 1$ for Debye/non-Debye cases) for each semicircular relaxation yielding information concerning the nature of the distribution of the relaxation time and the energy-depth/level of its constituting elements;

7. extrapolation of dc behavior (depending on the frequency range in the low-side measurement);

8. extrapolation of high-frequency dielectric (geometric) behavior (depending on the frequency range in the high-side measurement);

9. barrier-related parameters (such as flat-band potential or built-in-potential or the amount of band-bending, barrier height, depletion layer width or electrical thickness, carrier density, etc.);

10. trapping phenomena (when present) possessing energy depth, capture crosssection, capture-rate (measure of relaxation time), defect charge state (donor/ acceptor-like, neutral, etc.), etc; and

11. isolation of trapping (when present) contribution in the frequency or time domain;

12. phase angle $(\delta)$ between the voltage and current;

13. Bode magnitudes representing absolute values of the four complex plane formalisms (i.e., $\left|\mathrm{Z}^{*}\right|,\left|\mathrm{Y}^{*}\right|,\left|\mathrm{C}^{*}\right|$, and $\left|\mathbf{M}^{*}\right|$ );

14. Bode magnitudes representing values of the electrical parameters or quantities constituting the four complex plane formalisms (i.e., $Z^{\prime}, Z^{\prime \prime}, Y^{\prime}, Y^{\prime \prime}, C^{\prime}, C^{\prime \prime}, M^{\prime}$, and $M$ " given by equations 2-9 and 11-14);

15. Bode slope of each segment of the straight line (i.e., more than one slope for multiple segments in an extended frequency of measurement); and

16. inflection point between the successive straight line segments in the Bode representation indicating relaxation time.

Each of these parameters provides a meaningful information concerning the electrical behavior of the DUT, and can be used to construct an equivalent circuit 
model. The elements in this model can then be modified by changing processing variables and fabrication methods to optimize device performance.

\section{DISCUSSION}

The interpretation of an identified mechanism can be influenced by the nomenclature of the electrical parameters selected with or without incorporating physical geometrical factors. Therefore, it will be shown in a generic manner how the nomenclature containing microstructural features (excluding "inductive" effect $^{35}$ originating from DUT, electrode material, DUT/electrode configuration, etc.) can mislead the physical occurrence (or location) of the phenomena. These include some case examples and their implications on MPCHPH systems. The frequently used nomenclatures of the derived circuit elements (series or parallel equivalent) are as follows: (1) resistivity/conductivity or resistance/conductance, and (2) permittivity or capacitance.

Schematic illustrations depicted in Figures 5, 6, and 7 displaying immittance spectra of $\mathrm{ZnO}-\mathrm{Bi}_{2} \mathrm{O}_{3}$ based varistors are used to describe the advantage and/or purpose of the multi-plane representation. Each operative phenomenon is isolated from the total behavior and, thus, the lumped response is further delineated in Figure 5. Varistor is taken as an example because it represents a typical MPCHPH system. If Figure 6 is used at first, before exploiting Figures 5 and 7 with or without the resonating behavior at high frequencies, it is easy to provide a confusing interpretation. This is because $Z^{*}$-plane plot exhibits a severe lumped behavior than the other two plots in Figures 5 and 7 . The advantage of presenting these three schematics in a simultaneous fashion is that they display the same bulk dielectric behavior confirming resonating parameters.

\section{Case 1}

The "bulk phenomenon" of an MPCHPH system identified must indicate a frequency-independent characteristic. As a high-frequency response, the "bulk phenomenon" corresponds to an SCSJ-like dielectric behavior. The "bulk resistance" results from a homogeneous conduction process throughout the seriesparallel microstructural network of conducting paths. It is reasonable to assume a uniform distribution of electrical field stress as all junctions (i.e., grain-boundaries or intergranular regions) are shorted out at high frequencies. This means that the electrical barriers are no longer operative at high frequencies and, thus, leaving only "bulk phenomenon." Under this circumstance, the "bulk resistance" can easily be transformed to "bulk resistivity" using physical geometrical factors. The main criterion for this conversion is that the electrical conduction across the junctions in the microstructural network behaves like a single-crystal. A similar situation is experienced at extremely high-voltages or high-currents, where the electrical barriers across the grain-boundary junctions are completely in breakdown process and yield only single-crystal like lumped grain behavior. This situation is once again analogous to the single-crystal like dielectric response. These two physical situations (i.e., at high-frequencies and/or high-voltages/currents) neglect any influence 

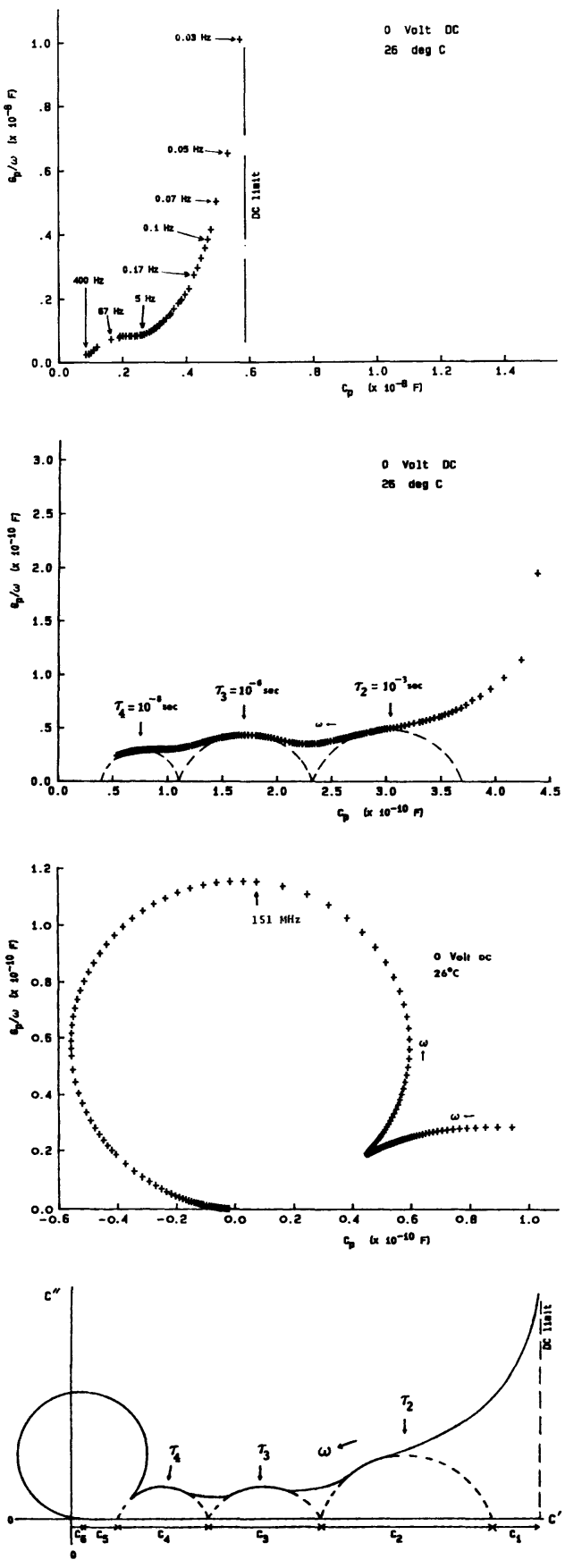

FIGURE 5 Capacitance $\left(\mathrm{C}^{*}=\mathrm{C}^{\prime}-j \mathrm{C}^{\prime \prime}=\mathrm{C}_{p}-j \frac{\mathrm{G}_{p}}{\omega}\right)$ plane representation of $\mathrm{ZnO}-\mathrm{Bi}_{2} \mathrm{O}_{3}$ based varistor at near room temperature in the approximate frequency range $(a) 10^{-2} \leq f \leq 400 \mathrm{~Hz},(b) 5 \leq$ $f \leq 13 \times 10^{6} \mathrm{~Hz},(c) 10^{6} \leq f \leq 10^{9} \mathrm{~Hz},(d)$ generic behavior in the $\mathrm{C}^{*}$-plane combining the behavior in (a), (b), and (c). 


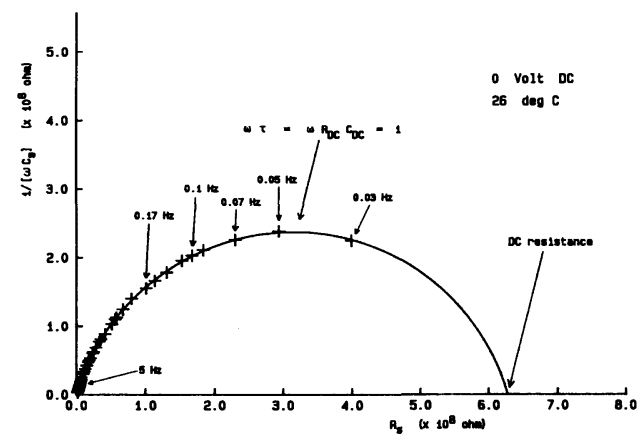

FIGURE 6 Impedance $\left(\mathrm{Z}^{*}=\mathrm{Z}^{\prime}-j \mathrm{Z}^{\prime \prime}=\mathrm{R}_{\mathrm{s}}-j 1 / \omega \mathrm{C}_{s}\right)$ plane representation of the $\mathrm{ZnO}_{\mathrm{n}}-\mathrm{Bi}_{2} \mathrm{O}_{3}$ based varistor at near room temperature in the approximate frequency range $10^{-2} \leq f \leq 10^{5} \mathrm{~Hz}$. The immittance data in the frequency range $10^{5}<f \leq 10^{9} \mathrm{~Hz}$ are not shown. The lumped grain dielectric behavior complemented by the left-intercept of this semicircular relaxation is obtained in the $\mathrm{C}^{*}$-plane of Figure 5(b) or 5(d).

of the disorder in the grain orientation as it may have no impact on the total electrical behavior. In the case of a sensitive DUT this issue should be considered. Thus, "bulk conductance" or "bulk conductivity" would bear a meaning identical to the "bulk resistance" or "bulk resistivity," respectively. Any of these four nomenclatures indicates the same mechanism and must yield the "average grain carrier density." The "average grain carrier density" assumes even distribution of carriers in each grain. In the same way, "bulk phenomenon" resolves the other four nomenclatures derived from "capacitance" when series and parallel configuration are considered.

Figures 5, 6, and 7 provide examples of "bulk phenomenon" corresponding to the high-frequency domain. Figure 6 has a tiny left-intercept on the left-side of the large semicircular relaxation in the $\mathrm{Z}^{*}$-plane. It is identified as a bulk resistance $\left(\mathrm{R}_{\mathrm{ZnO}} \approx\right.$ $10 \Omega$ ) on the real-axis attributing to the lumped $\mathrm{ZnO}$ grains' resistance. This situation has also been recognized in references 38 and 39. However, Figure 7

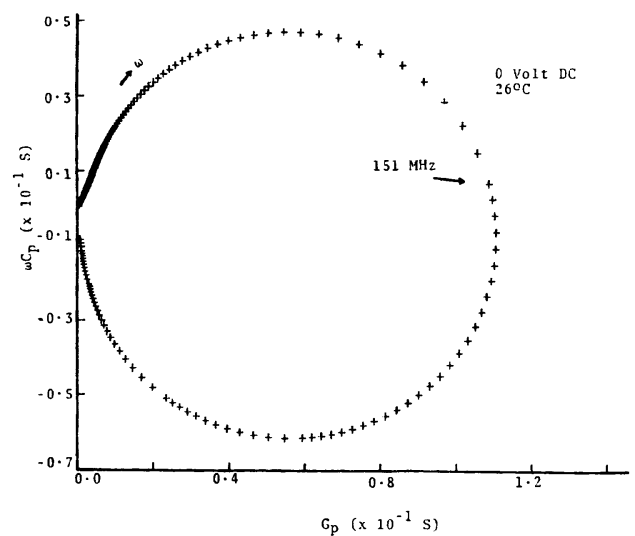

FIGURE 7 Admittance $\left(\mathrm{Y}^{*}=\mathrm{Y}^{\prime}+j \mathrm{Y}^{\prime}=\mathrm{G}_{\mathrm{p}}+j \omega \mathrm{C}_{\mathrm{p}}\right.$ ) plane representation of the $\mathrm{ZnO}-\mathrm{Bi}_{2} \mathrm{O}_{3}$ based varistor at near room temperature in the approximate frequency range $10^{6} \leq f \leq 10^{9} \mathrm{~Hz}$. The chord of the semicircle or circle passing through $x=0, y=0$ and parallel to $x$-axis yields the left-intercept value of $R_{\mathrm{ZnO}} \approx 10 \Omega$ obtained in Figure 6 . 
clarifies this situation when the chord of the circle coincides with the value obtained in Figure 6 for $R_{\mathrm{ZnO}}$. A detailed description on this parameter is provided in references 35 and 40 . Figure 5 supports the resolution of the bulk capacitance as the dielectric behavior $\left(\mathrm{C}_{\mathrm{ZnO}}=\mathrm{C}_{6}\right)$ of the lumped $\mathrm{ZnO}$ grains. The bulk resistance is obtained from the chord (passing through $x=0, y=0$ and parallel to $x$-axis) of the circle. This value is the same as the left-intercept obtained in Figure 6 . Thus, to describe bulk phenomenon and achieving complete information on the microstructural network of electrical paths, each of these illustrations is important. The bulk parameters resolved in Figures 5,6 , and 7 can be converted to bulk resistivity $\left(\rho_{\mathrm{ZnO}}\right)$ and bulk permittivity $\left(\epsilon_{\mathrm{ZnO}}\right)$ using physical geometrical factors.

From the $C^{*}$-plane plot of Figure 5, it is evident that the semicircular relaxation in the $\mathrm{Z}^{*}$-plane (Figure 6) cannot simply be designated as the grain-boundary phenomenon. The $Z^{*}$-plane relaxation yielded several other relaxations in the $\mathrm{C}^{*}$-plane. Figure 6 makes the situation complicated if the high-frequency resonance phenomenon is incorporated. That is why the resonance phenomenon ${ }^{3,22,35}$ is displayed separately as Figure 7. Although the left-intercept in Figure 6 is identified as a bulk resistance, two consecutive semicircles were not obvious as the resonance phenomenon intercepted the bulk dielectric behavior in ascribing the $\mathrm{Z}^{*}$-plane behavior. The bulk dielectric quantity $\mathrm{C}_{6}\left(\mathrm{C}_{\mathrm{ZnO}}\right)$ is obtained as a parallel parameter in Figure 5, which effectively is also in parallel with the bulk resistance, $\mathrm{R}_{\mathrm{ZnO}}$. Therefore, a precautionary measure is necessary before confirming a phenomenon of an electrical response only by inspecting the semicircular feature in the $\mathrm{Z}^{*}$-plane. The bulk phenomenon thus identified can be normalized to obtain the average carrier density in the grains for the $\mathrm{ZnO}-\mathrm{Bi}_{2} \mathrm{O}_{3}$ based varistors. This is analogous to the SCSJ-like situation and, therefore, a carrier density of about $10^{17} / \mathrm{cm}^{3}$ can be obtained. Same numerical value is also obtained from the post-nonohmic ohmic region $^{22,40}$ utilizing the current-voltage (I-V) behavior and optical method. ${ }^{41}$

\section{Case 2}

The identification of the grain-boundary resistance becomes obvious at a frequency range lower than that of the bulk resistance phenomenon. This is evident in Figure 6 by the chord of the semicircular relaxation. The grain-boundary resistance can simply be transformed to grain-boundary conductance. It cannot be converted to the corresponding resistivity or conductivity, because the grain-boundary region does not have any connection with the physical geometrical factors. The grainboundary phenomenon is associated with the intergranular region where two consecutive grains are in intimate contact (possibly with the presence of a second phase at the interfaces). This intimate contact can cause a potential barrier or discontinuity in the conducting path due to the charge storage and/or trapping at the defect sites (primarily originating from the dangling bonds). Each potential barrier constitutes a certain electrical thickness, corresponding to a transition or depletion region for a Schottky-like barrier. Since these regions in the microstructure are more resistive than the surrounding grains, a major portion of the applied electric field is dropped across these regions. Thus, the microstructural geometrical factors constitute contact area between the successive grains and electrical thickness 
of the grain-boundaries. These factors should be used in converting grain-boundary resistance to grain-boundary resistivity. Implying the same argument, grainboundary capacitance can be converted to grain-boundary permittivity. Thus, it becomes obvious that such a conversion is meaningful only when the grainboundary phenomenon is properly identified in the frequency-domain, and these two microstructural geometrical factors (contact area and electrical thickness) have nothing to do with the physical geometrical factors.

The grain-boundary relaxation yields a resistance of about $6.2 \times 10^{8} \Omega$ in Figure 6 . This response is extracted while the grain-boundary junctions are not electrically shorted. The grain-boundary barriers are still in effect in the frequency range $(f \leq$ $\sim 10^{6} \mathrm{~Hz}$ ). As the depletion region thickness and grain-to-grain contact area are not known, the grain boundary resistance cannot be converted or normalized to grain boundary resistivity. If single grain-boundary junctions are investigated, individual thickness of the depletion region and grain-to-grain contact area will be known. ${ }^{42}$

In the event when single-frequency (arbitrarily selected spot-frequency) immitance components are used in investigation, the nature of dispersion is not identified or the operative phenomena can only be assigned, mostly, by guessing. This is one of the reasons why single-frequency dielectric evaluation incorporating physical geometrical factors can cause confusion. Extending this concept further, until the term grain-boundary resistivity is ascertained, the term grain-boundary conductivity will not be appropriate to use. If a fraction of the contact area contributes to the total conduction process (due to the non-planer contact configuration across the grain-boundary interfaces), this contact area is better termed as the effective contact area. In order to understand the role of either contact area or effective contact area, a grain-to-grain single-junction study is helpful. ${ }^{42}$ The effective contact area can be determined using classical Mott-Schottky equation if the carrier concentration is spatially uniform in each grain, as demonstrated in the literature. ${ }^{22,43}$

\section{Case 3}

The electrode-related contribution to the immittance components can be observed at frequencies much lower than the frequency-range for the grain-boundary phenomenon. The contributions associated with each electrode terminal may not be identical, although their physical nature or origin is likely to be the same considering the degree of non-uniformity and relevant concentration of the dangling bonds at the DUT's surface. A mismatch in the work-function between the electrode material and the DUT is often responsible for the electrode-related contribution. Another source of electrode-related contribution, commonly observed in ionic or electrolytic materials, is ascribed to the charge accumulation or blocking of the charge carriers. For reasons similar to that of the grain-boundary phenomenon the electrical parameters associated with the electrode, once identified cannot be normalized using physical geometrical factors. Although the physical electrode area may be applied, the barrier layer thickness (i.e., electrical thickness) should be determined and used to replace the physical thickness of the DUT.

Another perspective of the electrode effect is the evolution of the resonance phenomena in certain material systems. This is observed, in general, at very high 
frequencies and can be seen in Figure 5. The resonance effect is not necessarily related to the piezoelectric resonance phenomena. Reference 35 describes combined (lumped) electrode-material effect for the observed resonance in Figure 5. When a material exhibits piezoelectric resonance, possible contribution from the electrode may not be separated. Therefore, electrode effects can be observed depending on the type of the material and the nature of investigation. It is, therefore, important to have sufficient knowledge of the type of materials investigated when a resonance phenomenon is observed.

\section{Case 4}

The trapping contribution to the terminal immittance is another important issue. This contribution causes dispersion of immittance with frequency. Figure 5 is an example of such a dispersion. For a lumped "grain-boundary phenomenon" the identity of trapping is completely masked as is seen in Figure 6. However, Figure 5 unravels the lumped response and several trapping contributions are evident. The traps are responsible for constituting electrical barriers at the grain-boundaries. They sustain these barriers under the applications of the ac small-signal amplitude in conjunction with the superimposed dc electric field. These traps may vary in their contributing R-C elements. Thus, the relaxation time (time constant) is sensitive to the frequency-domain measurements, and may vary or remain the same. Often these traps give rise to a lossy system. Depending on their nature of contribution to the total immittance components, the entire dispersion of immittance as a function of frequency may vary. The origin of the traps may be so different that the traps can respond at several frequency-domain steps for a wide range of frequency. This response can be masked by other simultaneously occuring phenomena (such as grain-boundary resistance and capacitance), especially in an MPCHPH system. Therefore, a full exploitation of the LP/CPA, BPA, and SA techniques (i.e., involving wide frequency-domain measurements and analyses) are essential to evaluate the trapping contribution(s). Although the traps are distributed all over an MPCHPH system, their frequency-domain response may become distinct when they actively respond from the regions where the electrical field drops significantly. The relaxation time is not affected when the physical geometrical factors are incorporated to its constituting elements. Thus, normalization of the constituting elements of the relaxation time

$$
\tau=\mathrm{RC}=\rho \epsilon
$$

is not essential. In other words, although trapping phenomenon is physically associated with certain physical or geometrical configuration, it does not require exact knowledge of the physical area and thickness to be precisely determined. However, for a left-interceptless single semicircular relaxation in the $\mathrm{Z}^{*}$-plane the normalization of $\mathrm{R}$ and $\mathrm{C}$ to obtain $\rho$ and $\epsilon$, respectively, will not affect the purpose.

Each trapping relaxation depicted in Figure 5 provides a pair of R-C series combination in the $\mathrm{C}^{*}$-plane. None of these parameters can be normalized, as their physical location is within the depletion region of the grain-boundary electrical 
barrier. $\tau_{2}$ through $\tau_{4}$ are trap-related relaxations. $\tau_{1}$ trapping is heavily distorted, and primarily influences the visibility of the semicircular relaxation in the $Z^{*}$-plane representation. This is attributed to the lossy nature of the $\mathrm{ZnO}$-based varistors. Extending the concept further, more lossy varistor will provide better visibility, possibly without sub- $\mathrm{Hz}$ measurement domain, in shaping the low-frequency arc of the semicircle in the $\mathrm{Z}^{*}$-plane. None of these traps can be ascribed to the physical geometrical factors. Since the relaxation time, $\tau$, is a geometry independent parameter, their constituting elements can be evaluated using non-equilibrium conditions (such as temperature, voltage, composition, processing variables, ambient gases, etc.). Exercising foregoing argument, there is no need of the state of normalization if $\tau$ and the constituting elements are systematically evaluated. ${ }^{44}$

The demonstration of the multi-plane analytical approach in Figures 5 and 6 provided a complete picture of the trap-controlled lumped grain-boundaries. Further, such an analysis demonstrated that the state of normalization of the as-measured immittance data is a confusing approach when extraneous contributing elements related to the traps exist in addition to the regular geometric contributions. Thus, the state of normalization cannot be used as a universal practice for the trap-related $\left(C_{1}\right.$ through $C_{4}$ in Figure 5) and trapless $\left(C_{5}\right.$ and $\left.C_{6}\right)$ parameters.

\section{Case 5}

The choice of plotting electrical quantities and their units is left to the investigator. A series of arbitrary units can be derived from a basic root unit. In the LP/CPA technique, $Z^{*}$ - or $\rho^{*}$-plane plot utilizes the unit of resistance (i.e., $\Omega$ ) and/or resistivity $(\Omega-\mathrm{cm})$, respectively. Sometimes $\Omega-\mathrm{cm}^{2}$ is also used to represent either of these two complex plane plots. The root unit $\Omega$ or normalized unit $\Omega$-cm (using physical geometrical factors) has a physical significance and are based on fundamental physical parameters, such as resistance or resistivity. Ascertaining the same fundamental cause, the physical significance of $\Omega-\mathrm{cm}^{2}$ is vague. It does not represent a unit of a scientific parameter or a physical quantity. It has no connection with the use of sheet resistivity for an SCSJ-like system. In this case either resistivity or conductivity is exploited through a dc conduction process, describing exclusively the in-phase (or real) quantity. This in-phase quantity is invariant whether series or parallel configuration is considered.

A derived unit has no physical meaning when it does not reflect the true nature of a physical quantity. Depending on the material system, a derived unit will have an impact on the interpretation whether series or parallel configuration is used in the data-analysis. The reactance of the impedance $\left(Z^{*}=R_{s}-1 / \omega C_{s}\right)$ can be converted to the equivalence of $\Omega-\mathrm{cm}^{2}$ using the physical electrode area in the series capacitance $\left(\mathrm{C}_{\mathrm{s}}\right)$ to represent capacitance per unit area. Since the unit of the axes is not impedance $\mathrm{Z}^{*}$ (or resistivity $\rho^{*}$ ) by definition, practicing such a plot is not worthwhile. To extend the argument further, $\Omega-\mathrm{cm}^{2}$ can be used as a hypothetical parameter, as sheet resistance is considered only under the dc $(f \rightarrow 0 \mathrm{~Hz})$ condition. At this conjencture, the significance of $C_{s}$ is totally diluted in comparison to that of $\mathrm{C}_{\mathrm{p}}$ or vice versa. 
To validate a derived unit (such as $\Omega-\mathrm{cm}^{2}$ ), it is necessary to understand the role of the series capacitance $\left(\mathrm{C}_{\mathrm{s}}\right)$ converted with the physical electrode area. The reactance component of the impedance $\left(1 / \omega C_{s}\right)$ is in series with the series resistance $\left(\mathrm{R}_{\mathrm{s}}\right)$ yielding a blocking configuration of the DUT as $f \rightarrow 0 \mathrm{~Hz}$. This situation can be explained with a left-interceptless (i.e., no left-intercept on the real-axis) single semicircular (Debye-like or non-Debye) relaxation which is represented by an equivalent circuit consisting of a parallel R-C combination ( $\mathrm{Z}^{*}$-plot in Figure 3). This R-C parallel circuit is open to conduction at $f \rightarrow 0 \mathrm{~Hz}$. capacitance per unit area without further explanation of the real component in the equivalent circuit. Despite such an explanation of the terminal or circuit capacitance (series or parallel form), there is no meaning of the real component with a unit $\Omega-\mathrm{cm}^{2}$. It has no physical significance regardless of the number of semicircular relaxations. This is because the real component is not a representative of the sheet resistivity or sheet resistance discussed earlier. In fact, the situation becomes slightly more complicated if multiple semicircular relaxations are observed. This type of behavior is very common in the polycrystalline materials. ${ }^{10}$ Further complication is expected if each relaxation or any of the relaxations is non-Debye. Although an equivalent circuit can be constructed, the elements therein will have no meaning (with respect to the microstructural features) when $\Omega-\mathrm{cm}^{2}$ is used except for the lumped dielectric behavior. Same argument applies to the analysis using BPA or SA technique with this kind of unit. Thus, a derived unit is not a generalized representation of the DUT, as it cannot explain the purpose and meaning of each equivalent circuit element concerning the network (through microstructures or media) of electrical paths.

It is useful to add that to represent complex electrical quantities of $\mathrm{Y}^{*}$ or $\mathrm{C}^{*}$ per unit area is more meaningful than that of $\mathrm{Z}^{*}$ or $\mathrm{M}^{*}$ per unit area. This is because, the parallel configuration parameters are directly related to the physical situations of the DUT. However, achieving R-C parallel circuit in a semicircular relaxation of the $Z^{*}$ or $\mathbf{M}^{*}$-plane plot may enlighten the argument. Again, this achievement does not justify the concept of "capacitance per unit area" on a series configuration for the as-measured data.

Figures 8 and 9 illustrate the advantage of multi-plane analytical configuration, plotted with the unit of the as-measured electrical parameters of the immittance function, for polycrystalline yttria. ${ }^{5}$ Similar illustration is also provided for calcium zirconate $^{6}$ in Figure 10 . These illustrations provide congruity within the three complex planes. However, only one equivalent circuit may be emphasized for each material at a specific experimental condition from the combined effect of these three plots. More than one equivalent circuit provides a better resolution when other dominating mechanisms cannot be delineated by one complex plane representation. Thus, multi-plane analytical approaches unravel simultaneously operative mechanisms and provide a reasonable concept on the equivalent circuit modeling. The multiple relaxation phenomena are not disturbed by the unit of the electrical parameter used during the data-acquisition process. Nevertheless, BPA and SA techniques are exploited to assure the same operative mechanisms and equivalent circuit model as obtained via the LP/CPA for these material systems.

Since normalized unit inherently possesses limitations in interpreting simultaneously operative phenomena representing multiple semicircular 

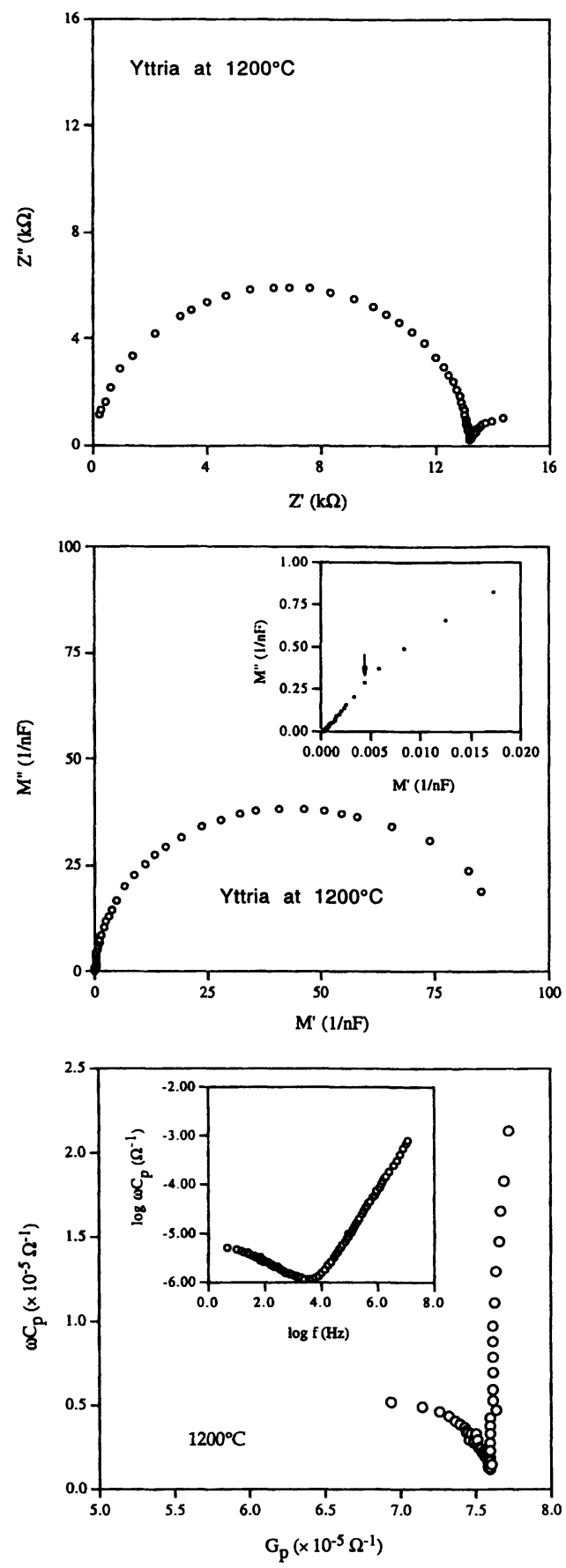

FIGURE 8 (a) Impedance plane representation of yttria at $1200^{\circ} \mathrm{C}$. (b) Modulus $\left(\mathbf{M}^{*}=\mathrm{M}^{\prime}+\right.$ $\left.j \mathrm{M}^{\prime \prime}=1 / \mathrm{C}_{s}+j \omega \mathrm{R}_{s}\right)$ plane representation of the same sample as in $(a)$. (c) Admittance plane representation of the same sample as in $(a)$ or $(b)$. The inset is a Bode plot showing variation of $\omega C_{p}$ as a function of frequency. The linear region in the high-frequency limit yields a value $C_{\infty}$ which causes vertical representation in $(c)$. 

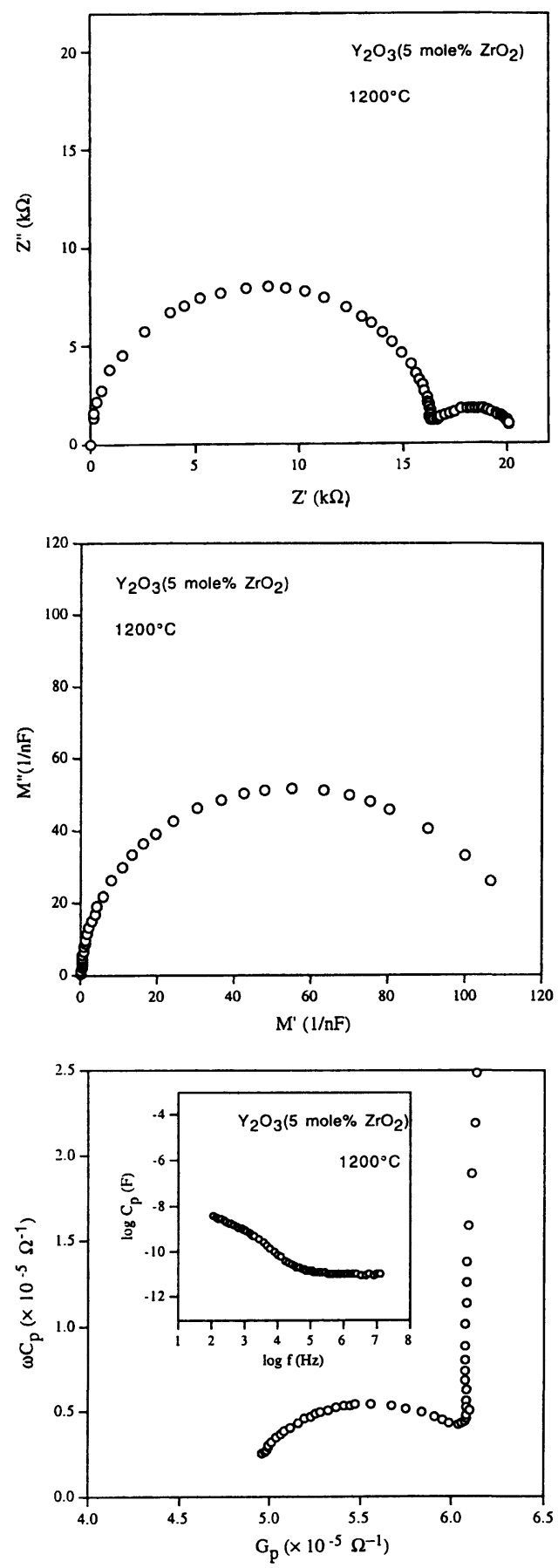

FIGURE 9 (a) Impedance plane representation for yttria doped with 5 mole \% zirconia sample at $1200^{\circ} \mathrm{C}$. (b) Modulus plane representation of the same sample as in $(a)$. (c) Admittance plane representation of the same sample as in $(a)$ or $(b)$. The inset is a Bode plot showing variation of $C_{p}$ as a function of frequency. The flat region in the high-frequency limit yields a value $\mathrm{C}_{\infty}$ which causes vertical representation in $(c)$. 

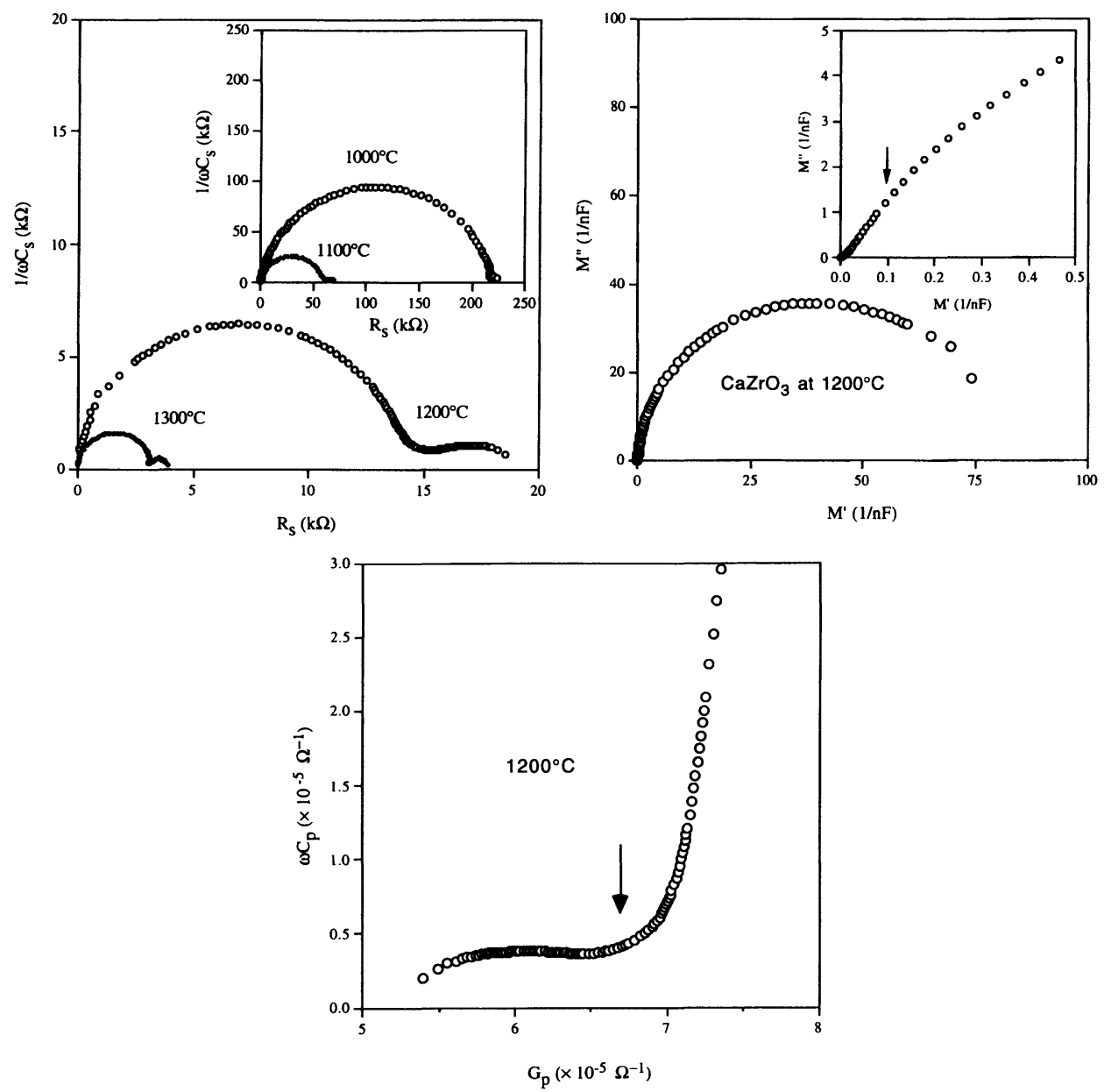

FIGURE 10 (a) Impedance plane representation of a calcium zirconate sample at four temperatures $\left(1000^{\circ} \mathrm{C}, 1100^{\circ} \mathrm{C}, 1200^{\circ} \mathrm{C}\right.$, and $\left.1300^{\circ} \mathrm{C}\right) .(b)$ Modulus plane representation of the same sample as in $(a)$ at $1200^{\circ} \mathrm{C}$. The inset highlights the demarcation with an arrow between high- and low-frequency responses documented in $(a)$. (c) Admittance plane representation of the same sample as in $(a)$ or $(b)$ at $1200^{\circ} \mathrm{C}$.

relaxations for the current or voltage dividing electrical paths, $\Omega$-cm (or similar unit of other electrical quantities/parameters constituting immittance function) should be used with caution. In the event when the as-measured data are used, freedom exists to choose whether multiple operative phenemena really require the state of normalization. Nevertheless, exploitation of the as-measured data are usually more meaningful than that of the normalized data in extracting and establishing "universal characteristics" for a DUT.

\section{CONCLUSIONS}

Various aspects of data handling/analyzing criteria for unknown material and device systems are emphasized. These systems include multi-phase/component 
heterogeneous polycrystalline hybrid materials, single-crystal/single-junction like divices, and electrochemical systems. In particular, the pitfalls of incorporating physical geometrical factors in the fragmented analytical procedure before ascertaining operative phenomena in conjunction with the nature of dispersion of immittance as a function of frequency are emphasized. The parameter bearing the sense of normalization is carefully identified. It is essential that the as-measured electrical data be examined thoroughly before incorporating physical geometrical factors into the electrical quantities or parameters of the immittance function. The data-handling criteria for a device under test are applicable to all types of material systems regardless of their structural configuration. Furthermore, systematic evaluation procedure and careful handling of the immittance data for engineering applications of these materials can provide a basis for microstructure-propertyprocessing relationships, which in turn can be used to optimize performance characteristics.

\section{ACKNOWLEDGMENTS}

It is author's pleasure to thank Dr. J. P. Dougherty (The Pennsylvania State University, State College, Pennsylvania 16802) and Dr. S. A. Akbar (The Ohio State University, Columbus, Ohio 43210) for useful suggestions, and Dr. C. C. Wang (The Ohio State University, Columbus, Ohio 43210) for stimulating discussion during the preparation of the manuscript. The author also appreciates interest and intellectual support of Mrs. Sanjida Khanam.

\section{REFERENCES}

1. (a) S. Trolier-McKinstry and R. E. Newnham; "Sensors, Actuators, and Smart Materials," MRS Bulletin, 18, 27-33 (1993). (b) R. E. Newnham and G. R. Ruschau; "Smart Electroceramics," J. Am. Ceram. Soc., 74, 463-480 (1991). (c) R. E. Newnham; "Composite Electroceramics," Ann. Rev. Mater. Sci., 16, 47-68 (1986).

2. M. A. Alim, S. Khanam, and M. A. Seitz; "Immittance Spectroscopy of Smart Components and Novel Devices," Act. Pass. Electron. Comp., 16, 153-170 (1994).

3. M. A. Alim; "Admittance-Frequency Response in Zinc Oxide Varistor Ceramics," J. Am. Ceram. Soc., 72, 28-32 (1989).

4. C. C. Chen, M. M. Nasrallah, H. U. Anderson, and M. A. Alim; "Immittance Response of $\mathrm{La}_{0.6} \mathrm{Sr}_{0.4} \mathrm{Co}_{0.2} \mathrm{Fe}_{0.8} \mathrm{O}_{3}$ Based Electrochemical Cells," J. Electrochem. Soc., 142, 491-496 (1995).

5. (a) C. C. Wang, V. D. Patton, S. A. Akbar, and M. A. Alim; "Effect of Zirconia Doping on the Electrical Behavior of Yttria," J. Mat. Res., 11, 422-429 (1996). (b) V. D. Patton, C. C. Wang, S. A. Akbar, and M. A. Alim; "The ac Electrical Behavior of Polycrystalline Yttria," J. Appl. Phys., 78, 1757-1762 (1995).

6. C. C. Wang, W. H. Chen, S. A. Akbar, and M. A. Alim; "High-Temperature ac Electrical Behavior of Polycrystalline Calcium Zirconate," submitted to J. Mat. Res. (1995).

7. (a) L. B. Younkman; "Development and Characterization of Ceramic-Based Carbon Monoxide Sensors," Ph.D. Dissertation, The Ohio State University, Columbus (June 1995). (b) A. M. Azad, S. A. Akbar, L. B. Younkman, and M. A. Alim; "High-Temperature Immittance Response in Anatase-Based Sensor Materials," J. Am. Ceram. Soc., 77, 3145-3152 (1994). (c) A. M. Azad, L. B. Younkman, S. A. Akbar, and M. A. Alim; "Characterization of $\mathrm{TiO}_{2}$-Based Sensor Materials by Immittance Spectroscopy," J. Am. Ceram. Soc., 77, 481-486 (1994).

8. A. K. Jonscher; "The 'Universal' Dielectric Response," Nature, 267, 673-679 (1977).

9. J. R. Macdonald; "Analysis of ac Conduction in Disordered Solids," J. Appl. Phys., 65, 4845-4853 (1989).

10. D. C. Sinclair and A. R. West; "Impedance and Modulus Spectroscopy of Semiconducting $\mathrm{BaTiO}_{3}$ Showing Positive Temperature Coefficient of Resistance," J. Appl. Phys., 66, 3850-3856 (1989).

11. W. Jakubowski and D. H. Whitmore; "Analysis of Electrolyte Polarization in Polycrystalline Sodium $\beta$ "-Alumina by a Complex Admittance Method," J. Am. Ceram. Soc., 62, 381-385 (1979). 
12. P. C. Searson, D. D. Macdonald, and L. M. Peter; "Frequency Domain Analysis of Photoprocesses at Illuminated Semiconductor Electrodes by Transient Transformation," J. Electrochem. Soc., 139, 2538-2543 (1992).

13. H. J. de Bruin and S. P. S. Badwal; "Faradaic Impedance Dispersion of the Noble-Metal/Ceramic Electrolyte Interface," J. Aust. Ceram. Soc., 14, 20-28 (1978).

14. T. M. Gur, I. D. Raistrick, and R. A. Huggins; "AC Admittance on Stabilized Zirconia with Porous Platinum Electrodes," Sol. St. Ion., 1, 251-271 (1980).

15. B. D. Cahan and C. T. Chen; "Questions on the Kinetics of $\mathrm{O}_{2}$ Evolution on Oxide-Covered Metals,” J. Electrochem. Soc., 129, 700-705 (1982).

16. E. A. Cooper, T. O. Mason, M. E. Biznek, and U. Balachandran; "Impedance Spectra During Solid-State Powder Reactions of Oxide Superconductors," J. Am. Ceram. Soc., 73, 154-157 (1990).

17. (a) M. A. Seitz, "Study of Heterogeneous Composite Materials via Lumped Parameter/Complex Plane Analysis," Int. J. Hybr. Microelectron., 3, 1-7 (1980); (b) J. J. Ackman and M. A. Seitz, "Methods of Complex Impedance Measurements in Biologic Tissue," CRC Crit. Rev. Biomed. Engr., 11, 281-311 (1984).

18. (a) D. L. Losee; “Admittance Spectroscopy of Deep Impurity Levels: ZnTe Schottky Barriers," Appl. Phys. Lett., 21 54-56 (1972). (b) D. L. Losee; “Admittance Spectroscopy of Impurity Levels in Schottky Barriers," J. Appl. Phys., 46, 2204-2214 (1975).

19. (a) G. Vincent, D. Bois, and P. Pinard; "Conductance and Capacitance Studies in GaP Schottky Barriers," J. Appl. Phys., 46, 5173-5178 (1975). (b) G. Vincent, A. Chantre, and D. Bois; "Electric Field Effect on the Thermal Emission of Traps in Semiconductor Junctions," J. Appl. Phys., 50, 5484-5487 (1979).

20. K. S. Cole and R. H. Cole; "Dispersion and Absorption in Dielectrics I: Alternating Current Characteristics," J. Chem. Phys., 9, 341-351 (1941).

21. F. A. Grant; "Use of Complex Conductivity in the Representation of Dielectric Phenomena," $J$. Appl. Phys., 29, 76-80 (1958).

22. M. A. Alim, M. A. Seitz, and R. W. Hirthe; "Complex Plane Analysis of Trapping Phenomena in Zinc Oxide Based Varistor Grain Boundaries,” J. Appl. Phys., 63, 2337-2345 (1988).

23. (a) Y. Shim and J. F. Cordaro, "Admittance Spectroscopy of Polycrystalline $\mathrm{ZnO}-\mathrm{Bi}_{2} \mathrm{O}_{3}$ and ZnO-BaO Systems," J. Am. Ceram. Soc., 71, 184-188 (1988); (b) J. F. Cordaro, Y. Shim, and J. E. May; "Bulk Electron Traps in Zinc Oxide Varistors," J. Appl. Phys., 60, 4186 (1986). (c) C. X. Qiu and I. Shih; "Investigation of Electrodeposited CuInSe ${ }_{2}$ Films by Admittance Spectroscopy," $J$. Appl. Phys., 64, 758-760 (1988). (d) L. V. Hmurcik and R. A. Serway, "Frequency Dispersion in the Admittance of the Polycrystalline $\mathrm{CU}_{2} \mathrm{~S} / \mathrm{CdS}$ Solar Cell," J. Appl. Phys., 61, 756-761 (1987). (e) K. Kobayashi, M. Takata, and Y. Fujimura, and S. Okamoto; "Investigation of Trapping States in a Nb-Doped Rutile by Admittance Spectroscopy," J. Appl. Phys., 60, 4191-4198 (1986). (f) M. D. Giulo, G. Micocci, and A. Tepore; "Admittance Spectroscopy of Traps in Au-InSe Schottky Cells," Sol. St. Elect., 27, 1015-1019 (1984). (g) P. Krispin; "Impurity Detection by Admittance-Frequency Dispersion," Phys. Stat. Sol. (a), 40, K81-K84 (1977).

24. H. W. Bode; Network Analysis and Feedback Amplifier Design, published by Van Nostrand, New York (1945).

25. D. V. Lang; "Deep-Level Transient Spectroscopy: A New Method to Characterize Traps in Semiconductors," J. Appl. Phys., 45, 3023-3032 (1974).

26. J. R. Macdonald; "Impedance Spectroscopy: Old Problems and New Developments," Electrochim. Acta, 35, 1483-1492 (1990).

27. M. A. Alim and S. A. Akbar; "Immittance Data Acquisition and Analytical Software Package," Developed at The Ohio State University, Columbus, Ohio (1992).

28. (a) D. W. Marquardt; "An Algorithm for Least-Squares Estimation of Nonlinear Parameters," $J$. Soc. Indust. Appl. Math. (Am. Math.), 11, 431-441 (1963). (b) K. Levenberg; "A Method for the Solution of Certain Non-Linear Problems in Least Squares," Quart. Appl. Math., 2, 164-168 (1944).

29. Y. T. Tsai and W. H. Whitmore, "Nonlinear Least-Squares Analyses of Complex Impedance and Admittance Data for Solid Electrolytes," Sol. St. Ion., 7, 129 (1982).

30. W. Schreiner, M. Kramer, S. Krischer, and Y. Langsam; "Nonlinear Least-Squares Fitting," PC Tech Journal (IBM), pp 170-185, May 1985.

31. (a) J. R. Macdonald; "Comparison and Application of Two Methods for the Least Squares Analysis of Immittance Data," Sol. St. Ion., 58, 97-107 (1992). (b) J. R. Macdonald and A. J. Garber; "Analysis of Impedance and Admittance Data for Solids and Liquids," J. Electrochem. Soc., 124, 1022-1030 (1977). (c) J. R. Macdonald, J. Schoonman, and A. P. Lehnen; "The Applicability and Power of Complex Nonlinear Least Squares for the Analysis of Impedance and Admittance Data," J. Electroanal. Chem., 131, 77-95 (1982).

32. R. D. Armstrong, M. F. Bell, and A. A. Metcalfe; "A Method for Automatic Impedance Measurement and Analysis," J. Electroanal. Chem., 77, 287-298 (1977). 
33. S. Bhatnagar, S. Gupta, and K. Shahi; "Automated Measurement of ac Impedance Measurement and Analysis," Sol. St. Ion., 31, 107-115 (1988).

34. (a) B. A. Boukamp; "Equivalent Circuit (EQUIVCRT.PAS)," User's Manual, Second Revised Edition, Report: CT88/265/128 and CT89/214/128, Department of Chemical Technology, University of Twente, P.O. Box 217, 7500 AE Enschede, Netherlands, May 1989. (b) B. A. Boukamp; “A Microcomputer Based System for Frequency Dependent Impedance/Admittance Measurements," Sol. St. Ion., 11, 339-346 (1984).

35. M. A. Alim; "High-Frequency Terminal Resonance in $\mathrm{ZnO}-\mathrm{Bi}_{2} \mathrm{O}_{3}$ Based Varistors," J. Appl. Phys., 74, 5850-5853 (1993).

36. (a) A. M. Azad, S. A. Akbar, S. G. Mhaisalkar, L. D. Birkefeld, and K. S. Goto; "Solid State Gas Sensors: A Review," J. Electrochem. Soc., 139, 3690-3704 (1992). (b) A. M. Azad, S. A. Akbar, and L. B. Younkman; "Ceramic Sensors for Carbon Monoxide and Hydrogen," Interface, pp 31-34, published by the Electrochemical Society, Pennington, N.J., December 1994.

37. (a) M. A. Alim; "Comment on "Temperature Dependence of Electrical Conductivity in Polycrystalline Tin Oxide'," J. Am. Ceram. Soc., 74, 1757-1758 (1991). (b) J. H. Lee, S. J. Park, and K. Hirota; "Reply to "Temperature Dependence of Electrical Conductivity in Polycrystalline Tin Oxide'," J. Am. Ceram. Soc., 74, 1759 (1991). (c) J. H. Lee, S. J. Park, and K. Hirota; "Temperature Dependence of Electrical Conductivity in Polycrystalline Tin Oxide," J. Am. Ceram. Soc., 73, 2771-2774 (1990).

38. (a) J. Fan and R. Freer; "The Roles Played by Ag and Al Dopants in Controlling the Electrical Properties of ZnO Varistors," J. Appl. Phys., 77, 4795-4800 (1995). (b) T. Asokan and R. Freer; "Grain and Grain Boundary Conduction in Zinc Oxide Varistors Before and After DC Degradation,” J. Europn. Ceram. Soc., 11, 545-550 (1993).

39. K. A. Abdullah, A. Bui, and A. Loubiere; "Low Frequency and Low Temperature Behavior of ZnO-Based Varistor by ac Impedance Measurements," J. Appl. Phys., 69, 4046-4052 (1991). (b) A Bui, K. A. Abdallah, A. Loubiere, M. Tao, and Q. C. Nguyen; "Impulse-Degradation Analysis of ZnO-Based Varistors by ac Impedance Measurements,” J. Phys. D: Appl. Phys., 24, 757-762 (1991).

40. (a) M. A. Alim and M. A. Seitz; "Singular Nature of Preferential Conducting Paths in ZnO-Based Varistors," J. Am. Ceram. Soc., 71, C246-C249 (1988). (b) M. A. Alim, M. A. Seitz, and R. W. Hirthe; "High-Temperature/Field Alternating-Current Behavior of ZnO-Based Varistors," J. Am. Ceram. Soc., 71, C52-C55 (1988).

41. L. M. Levinson and H. R. Philipp; "Optical Method for Determining the Grain Resistivity in ZnO-Based Ceramic Varistors," J. Appl. Phys., 47, 1112-1116 (1976).

42. (a) H. Wang, W. Li, and J. F. Cordaro; "Single Junctions in $\mathrm{ZnO}$ Varistors Studied by Current-Voltage Characteristics and Deep Level Transient Spectroscopy," Jpn. J. Appl. Phys., 34, 1765-1771 (1995). (b) H. Wang, W. A. Schulze, and J. F. Cordaro; "Averaging Effect on Current-Voltage Characteristics of ZnO Varistors," Jpn. J. Appl. Phys., 34, 2352-2358 (1995). (c) G. D. Mahan, L. M. Levinson and H. R. Philipp; "Single Grain Junction Studies of ZnO Varistors-Theory and Experiment," Appl. Phys. Lett., 33, 830-832 (1978). (d) E. Olsson; "Interfacial Microstructure in ZnO Varistor Materials," Ph.D. Dissertation, Chalmers University of Technology, Göteborg, Sweden (1988). (e) M. A. Seitz, A. K. Verma, and R. W. Hirthe; "AC Electrical Behavior of Individual MOV Grain Boundaries," Ceramic Transactions: Advances in Varistor technology, L. M. Levinson edited, Published by The American Ceramic Society, Westerville, Ohio, 3, 135-144 (1989). (f) M. A. Alim; unpublished work.

43. M. A. Alim; "An Analysis of the Mott-Schottky Behavior in $\mathrm{ZnO}-\mathrm{Bi}_{2} \mathrm{O}_{3}$ Based Varistors," J. Appl. Phys., 78, 4776-4779 (1995)

44. (a) M. A. Alim; "Influence of Intrinsic Trapping on the Performance Characteristics of $\mathrm{ZnO}-\mathrm{Bi}_{2} \mathrm{O}_{3}$ Based Varistors," Act. Pass. Electron. Comp. 17, 99-118 (1994). (b) M. A. Alim; "Influence of Multiple Trapping Phenomena on the Applications Criteria of $\mathrm{ZnO}-\mathrm{Bi}_{2} \mathrm{O}_{3}$ Based Varistors," Act. Pass. Electron. Comp., 17 57-66 (1994). (c) L. C. Sletson, M. E. Potter, and M. A. Alim; "Influence of Sintering Temperature on Intrinsic Trapping in Zinc Oxide-Based Varistors," J. Am. Ceram. Soc., 71, 909-913 (1988). 

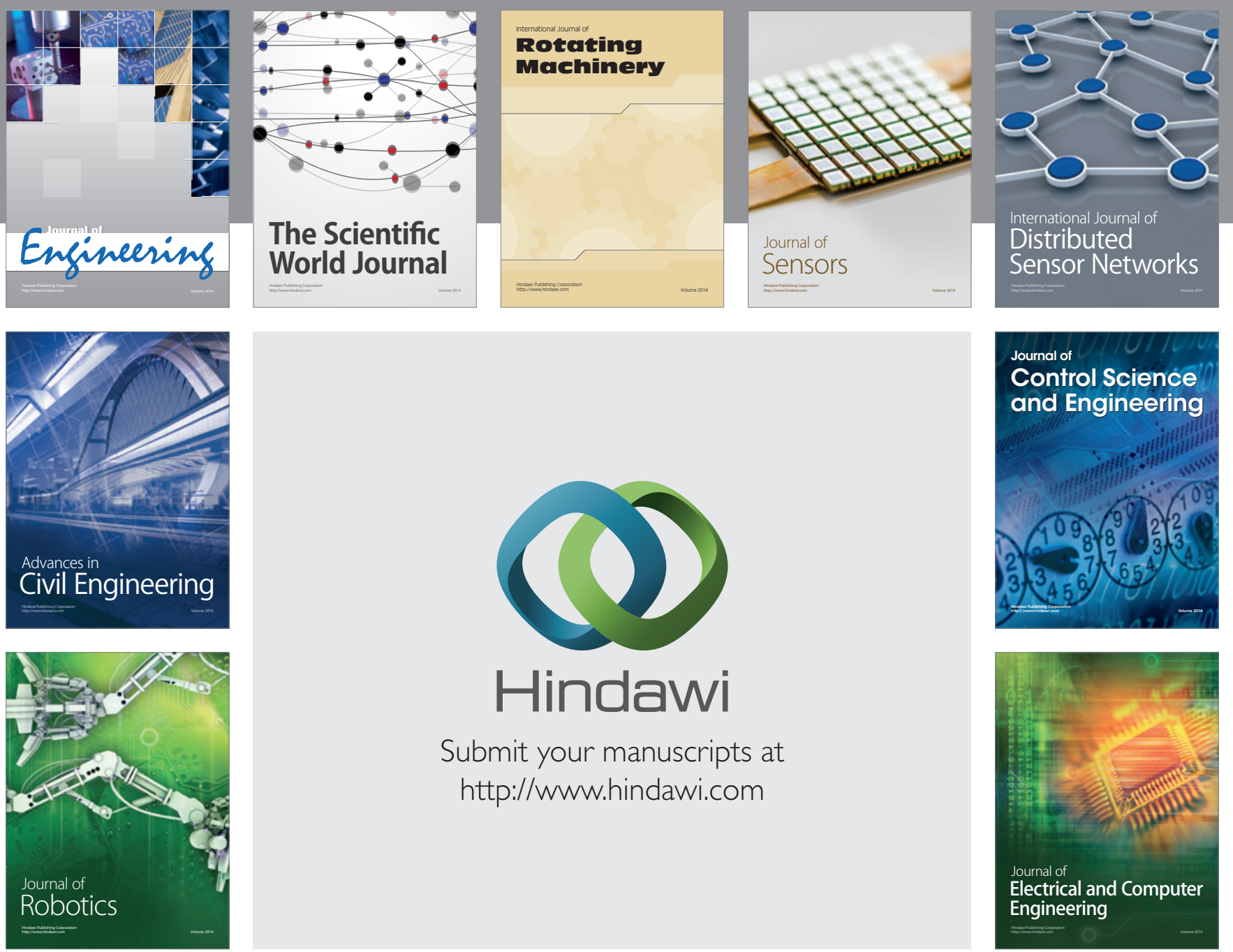

Submit your manuscripts at

http://www.hindawi.com
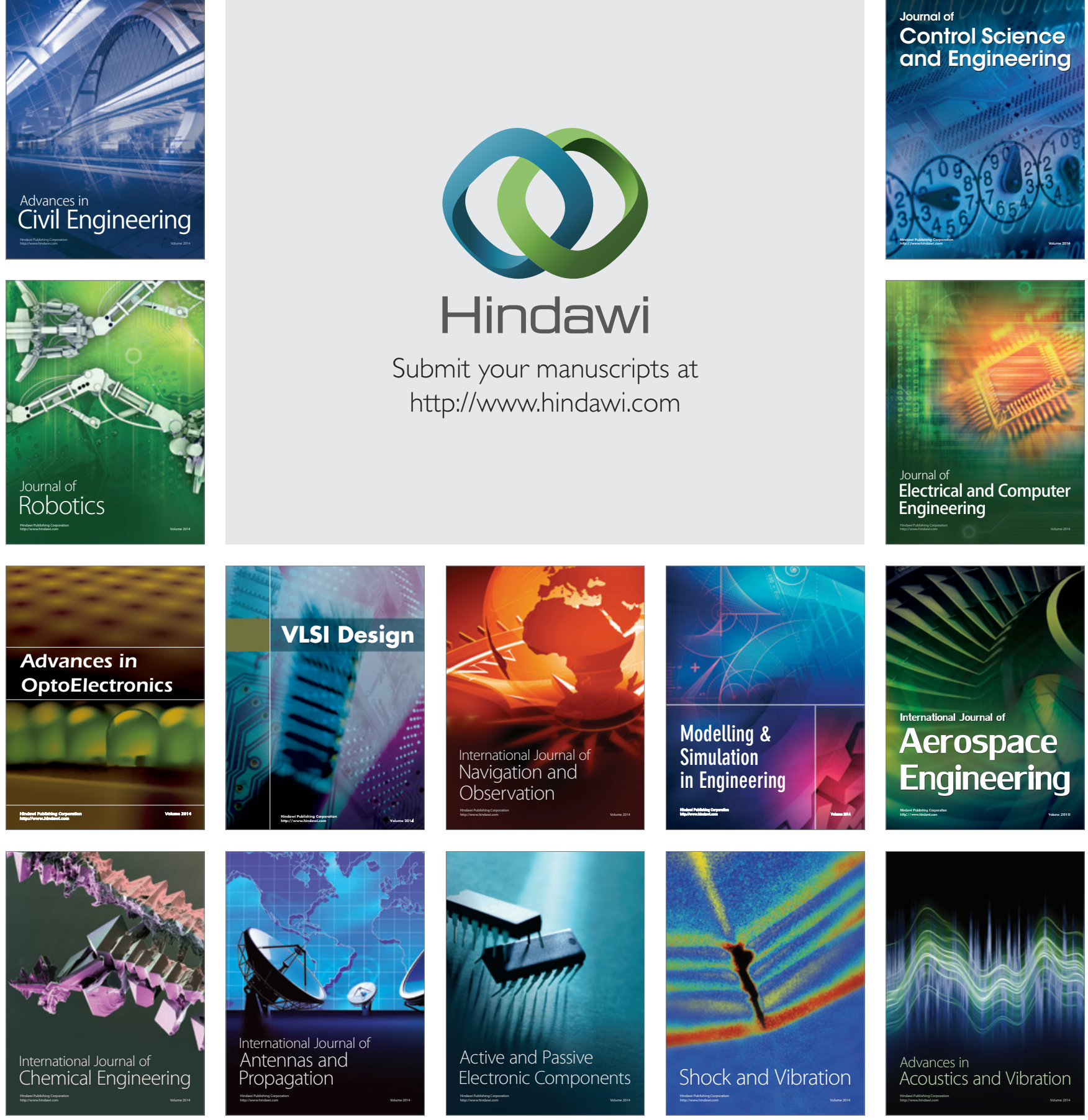